\title{
LOCAL AND GLOBAL EXISTENCE OF SOLUTIONS TO SEMILINEAR PARABOLIC INITIAL VALUE PROBLEMS
}

\author{
S. B. Cui (Cui Shangbin) \\ Department of Mathematics,Lanzhou University, \\ Lanzhou, Gansu 730000, People's Republic of China
}

\begin{abstract}
This paper is devoted to establishing local and global existence theorems for autonomous semilinear parabolic initial value problems. The local existence theorems do not require Lipchitz condition on nonlinear term. The global existence theorem is an extension of the well-known result of Fujita-Weissler for semilinear heat equations to general autonomous semilinear parabolic equations and systems.

Key words and phrases: Semilinear parabolic system, initial value problem, solution, local existence, global existence.
\end{abstract}

AMS 1991 subject classification number: $35 \mathrm{~K} 45$.

\section{Introduction}

In this paper we study local and global existence of solutions to the initial value problem

$$
\left\{\begin{array}{l}
\partial_{t} u=\sum_{|\alpha|=m} A_{\alpha} \partial_{x}^{\alpha} u+F\left(u, \partial_{x} u, \cdots, \partial_{x}^{m-1} u\right), \quad x \in R^{N}, t>0 \\
u(x, 0)=\varphi(x), \quad x \in R^{N}
\end{array}\right.
$$

where $m$ is an integer greater than $1, u=\left(u_{1}, u_{2}, \cdots, u_{n}\right), F=\left(F_{1}, F_{2}, \cdots, F_{n}\right)$ and $\varphi=$ $\left(\varphi_{1}, \varphi_{2}, \cdots, \varphi_{n}\right)$ are $n$-dimensional real (resp. complex) vector functions, $A_{\alpha}$ 's are constant real (resp. complex) $n \times n$ matrices, $\alpha$ 's represent indices in $Z_{+}^{N}$, and $\partial_{x}^{k} u$ represents the set of functions $\left\{\partial_{x}^{\alpha} u_{1}, \partial_{x}^{\alpha} u_{2}, \cdots, \partial_{x}^{\alpha} u_{n}:|\alpha|=k\right\}$, which we regard as a vector function. We are only concerned with parabolic problems, i.e., if we denote by $\Lambda(\zeta)\left(\zeta \in C^{N}\right)$ the maximum of real parts of all eigenvalues of the matrix $\sum_{|\alpha|=m}(i \zeta)^{\alpha} A_{\alpha}$, then it satisfies the following assumption:

$\left(A_{1}\right)$ there exist positive constants $a$ and $b$ such that

$$
\Lambda(\zeta) \leq-a|\operatorname{Re} \zeta|^{m}+b|\operatorname{Im} \zeta|^{m}, \quad \forall \zeta \in C^{N} .
$$

(Remark: As one can easily verify, the above assumption implies that $m$ is an even number.) 
A typical example is the following reaction-diffusion-convection system:

$$
\partial_{t} u=A \triangle u+F\left(u, \partial_{x} u\right), \quad x \in R^{N}, t>0,
$$

where $A$ is a diagonal matrix with positive diagonal elements. Another example is as follows: $m=2 l, n=1$ and

$$
\sum_{|\alpha|=m} A_{\alpha} \partial_{x}^{\alpha}=(-1)^{l-1} \triangle^{l} .
$$

It is easy to verify that (1.2) is satisfied by both of the above examples.

The first major topic of this paper is concerned with local existence of solutions to (1.1). As is well-known, under the assumption that nonlinear lower-order terms satisfy Lipchitz condition, local existence of solutions to semilinear evolution equations can be obtained rather widespreadly. In fact, such results have been proved for almost all the semilinear evolution equations whose linear principal parts are well-posed (c.f. $[1,2]$ for instance). A natural as well as important problem is whether local existence of solutions can be obtained under weaker conditions such as the nonlinear lower-order terms are only continuous. For nonlinear ordinary differential equations and systems, such result, called Peano's theorem, is well-known (c.f. [1, Theorem 4, p.4] and [3, Theorem 1, Chapter 4]). For semilinear parabolic equations and systems, we can use the theory of semilinear evolution equations associated to compact $C_{0}$ semigroup established by Pazy [4] and Lightbourne and Martin [5] to get similar results for their initial-boundary value problems on bounded domains and periodic initial value problems (c.f. also [2, Section 6.2 and Section 8.2]. However, as far as general initial value problems are concerned, since the corresponding semigroups are not compact, this theory is no longer applicable. Our first part of the main results fills partially in this gap. By following a different approach, we prove directly (without applying the semigroup theory) that under the assumption $\left(A_{1}\right)$, if $F$ is continuous, not required to satisfy Lipchitz condition, then for any $\varphi \in C_{B U}^{m-1}\left(R^{N}\right)$ (see the following section for this notation) (1.1) has a local weak solution; if in addition $F$ satisfies the assumption

$\left(A_{2}\right)$ for any $M>0$ there exists a corresponding consatant $C_{M}>0$ such that

$$
\left|F\left(w_{0}, w_{1}, \cdots, w_{m-1}\right)\right| \leq C_{M}\left(\left|w_{0}\right|+\left|w_{1}\right|+\cdots+\left|w_{m-1}\right|\right)
$$

for all $\left(w_{0}, w_{1}, \cdots, w_{m-1}\right)$ satisfying $\left|w_{j}\right| \leq M(j=0,1, \cdots, m-1)$,

then for any $\varphi$ belonging to $W^{m-1,1}\left(R^{N}\right) \cap C_{B U}^{m-1}\left(R^{N}\right)$, (1.1) has a local strong solution. When $F$ is locally Hölder continuous, these solutions are naturally classical solutions.

The second major topic of this paper is concerned with global existence of solutions. We prove that if $F$ is continuous and satisfies the assumption

$\left(A_{3}\right)$ there exist constants $C>0, M>0$ and $p_{j}>1+(m-j) /(N+j)(j=0,1, \cdots$, $m-1$ ) such that

$$
\left|F\left(w_{0}, w_{1}, \cdots, w_{m-1}\right)\right| \leq C\left(\left|w_{0}\right|^{p_{0}}+\left|w_{1}\right|^{p_{1}}+\cdots+\left|w_{m-1}\right|^{p_{m-1}}\right)
$$


for all $\left(w_{0}, w_{1}, \cdots, w_{m-1}\right)$ satisfying $\left|w_{j}\right| \leq M(j=0,1, \cdots, m-1)$;

then there exists $\delta>0$ such that for any $\varphi \in W^{m-1,1}\left(R^{N}\right) \cap C_{B U}^{m-1}\left(R^{N}\right)$ satisfying

$$
\|\varphi\|_{W^{m-1,1}\left(R^{N}\right)}+\|\varphi\|_{W^{m-1, \infty}\left(R^{N}\right)} \leq \delta
$$

the problem (1.1) has a global strong solution which decays to zero as $t \rightarrow \infty$.

The problem of global existence of solutions to nonlinear evolution equations and systems has drawn wide interest since 1960's when it was found that solutions to certain nonlinear evolution equations did not exist globally and, more remarkablly, that global existence of solutions were crucially influenced by the space dimension (c.f. [6-14] and the references cited therein). Related to the problem (1.1), we mention here the literatures of Fujita [7], Kobayashi et al [8], Weissler [9], Ponce [10] and Zheng [11]. By Fujita [7], Kobayashi et al [8] and Weissler [9], the problem

$$
\left\{\begin{array}{l}
\partial_{t} u=\Delta u+u^{p}, \quad \text { in } R^{N} \times(0, \infty) \\
u=\varphi, \quad \text { on } R^{N}
\end{array}\right.
$$

has no global solution for any nontrivial nonnegative $\varphi$ provided $1<p \leq 1+2 / N$, while it has such solution for small nonnegative $\varphi$ provided $p>1+2 / N$. The literature of Ponce [10] extends, among other contributions, the positve part of the above results to the fully nonlinear equation

$$
\partial_{t} u=\triangle u+f\left(u, \partial_{x} u, \partial_{x}^{2} u\right) .
$$

He proves that if $f\left(u, w_{1}, w_{2}\right)$ is sufficiently smooth (e.g. of $C^{2}$-class) and satisfies the condition

$$
\left|f\left(u, w_{1}, w_{2}\right)\right| \leq C\left(|u|+\left|w_{1}\right|+\left|w_{2}\right|\right)^{p}, \quad p>1+2 / N
$$

for small $\left(u, w_{1}, w_{2}\right)$, then the corresponding initial value problem has global solutions for sufficiently small initial data. When the function $f$ does not apparently depends on $u$, namely, $f\left(u, w_{1}, w_{2}\right)=f\left(w_{1}, w_{2}\right)$, Zheng [11] proves that the above condition can be replaced by the following weaker one:

$$
\left|f\left(w_{1}, w_{2}\right)\right| \leq C\left(\left|w_{1}\right|+\left|w_{2}\right|\right)^{2} .
$$

(Remark: We note that the condition (1.7) combined with the condition that $f$ is sufficiently smooth implies $p \geq 2$ ). These results have recently been systematically generalized to general autonomous nonlinear parabolic systems ([15-17]).

Restricting both the result of G. Ponce [10] and that of the present paper to the semilinear reaction-diffusion-convection equation

$$
\partial_{t} u=\triangle u+f\left(u, \partial_{x} u\right),
$$


we see that the structure condition on $f$ imposed in this paper is weaker than in G. Ponce [10]. Indeed, the assumption $\left(A_{3}\right)$ now reads as follows: for $|u| \leq M$ and $\left|w_{1}\right| \leq M$,

$$
\left|f\left(u, w_{1}\right)\right| \leq C\left(|u|^{p}+\left|w_{1}\right|^{q}\right), \quad p>1+2 / N, \quad q>1+1 /(N+1),
$$

which is obviously weaker than (1.7) applied to the function $f\left(u, w_{1}, w_{2}\right)=f\left(u, w_{1}\right)$. We believe, though unable to prove, that the condition $q>1+1 /(N+1)$ can not be weakened.

Although this paper is presented in a rather general skeleton, it is primarily motivated by and focused on the equation (1.8) and the system (1.3), which have not been studied sufficiently up to the present. From our results we see that in order to get a better understanding to these equation and system the divergence term $\partial_{x} u$ should not be treated simply like an additional $u$; instead, it should be handled, to certain extent, separately. Other related problems concerning (1.8) are studied in [12-14, 18, 19].

We point out that though the assumption $\left(A_{3}\right)$ for global existence of solutions with small initial data seems to be unweakenable for scalar equations, it is not optimal for systems (c.f. [20-21]). However, the results obtained in this paper are basic for more delicate analysis to the corresponding problems concerning systems (c.f. [21] for the example).

Let us now give an example of applications of our results. Consider the equation

$$
\partial_{t} u=\triangle u+u^{p}|\nabla u|^{q}
$$

where $p>0, q>0$. Applying Young's inequality, we get

$$
u^{p}|\nabla u|^{q} \leq \lambda u^{\frac{p}{\lambda}}+(1-\lambda)|\nabla u|^{\frac{q}{1-\lambda}}, \quad u \geq 0, \quad 0<\lambda<1 .
$$

In order to have $p / \lambda>1+2 / N$ and $q /(1-\lambda)>1+1 /(N+1)$ for some $\lambda \in(0,1)$, it is necessary and sufficient that $p$ and $q$ satisfy

$$
N(N+2) p+(N+1)^{2} q>(N+1)(N+2) .
$$

Therefore, if (1.10) is satisfied then by our results the initial value problem of (1.9) has global solutions for sufficiently small nonnegative initial functions. We note that neither $p$ nor $q$ is required to be greater than 1 because no Lipchitz condition is needed.

The layout of the remaining parts of this paper is as follows. In the following section we illustrate some essential notation and give the statement of our main results. Section III is devoted to deducing decay and continuity-modulus estimates for the solutions to the corresponding homogeneous linear problem. Section IV is devoted to deducing similar estimates for the solid potential integral associated to the operator $\partial_{t}-P\left(\partial_{x}\right)$. In Section $\mathrm{V}$ we present the proofs of local existence theorems (Theorems 2.1 and 2.2). The last section, VI, is arranged to give the proof of global existence theorem (Theorem 2.3). 


\section{Notation and Main Results}

Before stating the main results, let us introduce some notation. We use $L^{p}\left(R^{N}\right)(1 \leq$ $p \leq \infty)$ to denote both the scalar $L^{p}$ space and the $N$-dimensional vector $L^{p}$ space on $R^{N}$, and similarly for $W^{k, p}\left(R^{N}\right)\left(k \in Z_{+}, 1 \leq p \leq \infty\right), C_{0}^{\infty}\left(R^{N}\right)$ and $S\left(R^{N}\right)$ (which, evidently, does not produce confusion). The norm on the space $L^{p}\left(R^{N}\right)(1 \leq p \leq \infty)$ is writen as $\|\cdot\|_{p}$, and that on the space $W^{k, p}\left(R^{N}\right)\left(k \in Z_{+}, 1 \leq p \leq \infty\right)$ is writen as $\|\cdot\|_{k, p}$. We also use the following notation:

$$
W^{\infty, p}\left(R^{N}\right)=\bigcap_{k=0}^{\infty} W^{k, p}\left(R^{N}\right) \quad(1 \leq p \leq \infty) .
$$

The notation $C_{B U}\left(R^{N}\right)$ denotes both the space of bounded, uniformly continuous scalar functions on $R^{N}$ and the space of bounded, uniformly continuous $N$-dimensional vector functions on $R^{N}$. The notation $C_{B U}^{k}\left(R^{N}\right)\left(k \in Z_{+}\right)$has similar meaning. We use the notation $C_{B U}^{k+\theta}\left(R^{N}\right)\left(k \in Z_{+}, \theta \in(0,1)\right)$ to denot both the scalar Hölder space and the $N$-dimensional vector Hölder space on $R^{N}$. For a given positive integer $m$, we denote by $C_{B U}^{m *}\left(R^{N}\right)$ both the scalar and the $N$-dimensional vector Zygmund space, i.e., $\varphi \in C_{B U}^{m *}\left(R^{N}\right)$ if and only if $\varphi \in C_{B U}^{m-1}\left(R^{N}\right)$ and

$$
\sup _{x, y \in R^{N}} \sum_{|\alpha|=m-1} \frac{\left|\partial^{\alpha} \varphi(x+y)+\partial^{\alpha} \varphi(x-y)-2 \partial^{\alpha} \varphi(x)\right|}{|y|}<\infty .
$$

Besides, we identify a function $u(x, t)$ in two variables $x$ and $t$ with a function in one variable $t$ with values taken in some function space on $R^{N}$, and often simply write as $u(t)$. For a given function space $B$ on $R^{N}$, we use the notation $C([a, b) ; B)($ resp. $C([a, b] ; B), C((a, b)$; $B)$ ) to denote the set of continuous functions from $[a, b)$ (resp. $[a, b],(a, b))$ to $B$, and $L_{l o c}^{p}([a$, $b) ; B)\left(\right.$ resp. $\left.W_{l o c}^{1, p}([a, b) ; B)\right)$ to denote the set of measurable (resp. weakly differentiable) functions from $[a, b)$ to $B$ such that for any $c \in(a, b)$,

$$
\int_{a}^{c}\|u(t)\|_{B}^{p} \mathrm{~d} t<\infty \quad\left(\text { resp. } \int_{a}^{c}\left\{\|u(t)\|_{B}^{p}+\left\|u^{\prime}(t)\right\|_{B}^{p}\right\} \mathrm{d} t<\infty\right) .
$$

For $\theta \in(0,1)$, the notation $C^{\theta}([a, b] ; B)$ (resp. $\left.C^{\theta}((a, b) ; B), C^{\theta}((a, b] ; B)\right)$ denotes the space of uniformly (resp. locally) $\theta$-th order Hölder continuous functions from $[a, b]$ (resp. $[a, b),(a, b])$ to $B$. The notation $C^{1 \#}([a, b) ; B)$ (resp. $\left.C^{1 \#}((a, b) ; B)\right)$ represents the space of continuous functions $u$ from $[a, b)$ (resp. $(a, b)$ ) to $B$ such that for any $b^{\prime} \in(a, b)$ (resp. for any $\left.a^{\prime}, b^{\prime} \in(a, b), a^{\prime}<b^{\prime}\right)$ there exists a corresponding constant $C>0$ such that

$$
\left\|u(t)-u\left(t^{\prime}\right)\right\|_{B} \leq-C\left|t-t^{\prime}\right| \ln \left|t-t^{\prime}\right|, \quad \forall t, t^{\prime} \in\left(a, b^{\prime}\right)\left(\operatorname{resp} .\left(a^{\prime} b^{\prime}\right)\right)\left(0<\left|t-t^{\prime}\right| \leq \frac{1}{\mathrm{e}}\right),
$$

namely, $u \in C^{1 \#}([a, b) ; B)$ (resp. $\left.C^{1 \#}((a, b) ; B)\right)$ if and only if $u$ is locally $t \ln (1 / t)-$ Dini continuous on $[a, b)$ (resp. $(a, b))$. 
For a given function $\varphi \in L^{p}\left(R^{N}\right)(1 \leq p \leq \infty)$, the function $u(x, t)$ on $R^{N} \times[0, T)(T>$ 0 ) is said to be a weak solution of the problem (1.1) if it satisfies the following two conditions:

(i) $u \in C\left([0, T) ; L^{p}\left(R^{N}\right) \cap L_{l o c}^{1}\left((0, T) ; W^{m-1, p}\left(R^{N}\right)\right.\right.$;

(ii) $u$ satisfies the equations in (1.1) on $R^{N} \times(0, T)$ in distribution sense, and $u(0)=\varphi$. $u$ is said to be a strong solution of (1.1) if it satisfies the following stronger conditions:

(iii) $u \in C\left([0, T) ; L^{p}\left(R^{N}\right)\right) \cap L_{l o c}^{1}\left((0, T) ; W_{l o c}^{m, 1}\left(R^{N}\right)\right) \cap W_{l o c}^{1,1}\left((0, T) ; L_{l o c}^{1}\left(R^{N}\right)\right)$;

(iv) $u$ satisfies the equations in (1.1) a.e. in $R^{N} \times(0, T)$ and $u(0)=\varphi$.

Now we are able to state our first two main results.

Theorem 2.1 Suppose that the assumption $\left(A_{1}\right)$ is satisfied. Suppose furthermore that $F$ is continuous. Then for any $\varphi \in C_{B U}^{m-1}\left(R^{N}\right)$, there exists a corresponding $T^{*}>0$ such that the problem (1.1) has a weak solution $u$ on $R^{N} \times\left[0, T^{*}\right)$ which belongs to

$$
C\left(\left[0, T^{*}\right) ; C_{B U}^{m-1}\left(R^{N}\right)\right) \cap L_{l o c}^{\infty}\left(\left(0, T^{*}\right) ; C_{B U}^{m *}\left(R^{N}\right)\right) \cap C^{1 \#}\left(\left(0, T^{*}\right) ; C_{B U}\left(R^{N}\right)\right) .
$$

Moreover, if we denote by the same notation the supremum of all such $T^{*}$, then either $T^{*}=\infty$ or $T^{*}<\infty$ and

$$
\lim _{t \rightarrow T^{*}-0}\|u(t)\|_{m-1, \infty}=\infty
$$

Theorem 2.2 Suppose that the assumption $\left(A_{1}\right)$ is satisfied. Suppose furthermore that $F$ is continuous and satisfies the assumption $\left(A_{2}\right)$. Then for any $\varphi \in W^{m-1,1}\left(R^{N}\right) \cap$ $C_{B U}^{m-1}\left(R^{N}\right)$, there exists a corresponding $T^{*}>0$ such that the problem (1.1) has a strong solution $u$ on $R^{N} \times\left[0, T^{*}\right)$ which belongs to

$$
\begin{aligned}
& C\left(\left[0, T^{*}\right) ; W^{m-1,1}\left(R^{N}\right) \cap C_{B U}^{m-1}\left(R^{N}\right)\right) \cap L_{l o c}^{p}\left(\left[0, T^{*}\right) ; W^{m, p}\left(R^{N}\right)\right) \\
& \cap W_{l o c}^{1, p}\left(\left[0, T^{*}\right) ; L^{p}\left(R^{N}\right)\right), \quad \forall p \in(1, m] .
\end{aligned}
$$

Moreover, if we denote by the same notation the supremum of all such $T^{*}$, then either $T^{*}=\infty$ or $T^{*}<\infty$ and

$$
\lim _{t \rightarrow T^{*}-0}\left\{\|u(t)\|_{m-1,1}+\|u(t)\|_{m-1, \infty}\right\}=\infty .
$$

To give a clear expression of the next main result, we assume that in addition to the assumption $\left(A_{3}\right)$ the following conditions are satisfied:

$$
p_{j} \leq 1+\frac{m-j}{N+j-m} \quad \text { for } j>m-N
$$

This does not in essence strengthen the assumption $\left(A_{3}\right)$ because the condition $(2.3)$ is compatiple with the condition $p_{j}>1+(m-j) /(N+j) \quad(j=0,1, \cdots, m-1)$; if $(2.3)$ is 
not satisfied by a $p_{j}$ then we can substitute it with another one satisfing (2.3). Let

$$
\gamma=\min _{0 \leq j \leq m-1}\left(\frac{(N+j) p_{j}-N}{m}\right),
$$

and define a number $1 \leq p_{j}^{*} \leq \infty$ for every $j=0,1, \cdots, m-1$ as follows:

$$
p_{j}^{*}=\left\{\begin{array}{l}
\infty, \quad \text { if } N-m \gamma+j \leq 0 \\
N /(N-m \gamma+j), \quad \text { if } N-m \gamma+j>0
\end{array}\right.
$$

It is not difficult to verify that $1<\gamma<N /(N-1)$ and, consequently, $p_{j}^{*}>p_{j}$ for every $j=0,1, \cdots, m-1$.

Our another main result is the following

Theorem 2.3 Suppose that the assumptions $\left(A_{1}\right)$ is satisfiesd. Suppose that $F$ is continuous and satisfies the assumption $\left(A_{3}\right)$. Then there exists $\delta>0$ such that for any $\varphi \in W^{m-1,1}\left(R^{N}\right) \cap C_{B U}^{m-1}\left(R^{N}\right)$ satisfying

$$
\|\varphi\|_{m-1,1}+\|\varphi\|_{m-1, \infty} \leq \delta
$$

the problem (1.1) has a strong solution $u$ on $R^{N} \times[0, \infty)$ which belongs to

$$
\begin{aligned}
& C\left([0, \infty) ; W^{m-1,1}\left(R^{N}\right) \cap C_{B U}^{m-1}\left(R^{N}\right)\right) \cap L_{l o c}^{p}\left([0, \infty) ; W^{m, p}\left(R^{N}\right)\right) \\
& \cap W_{l o c}^{1, p}\left([0, \infty) ; L^{p}\left(R^{N}\right)\right), \quad \forall p \in(1, m] .
\end{aligned}
$$

Moreover, this solution possesses the following decay estimates:

$$
\begin{aligned}
\left\|\partial^{k} u(t)\right\|_{p} \leq & \left\{\begin{array}{l}
C(1+t)^{-\frac{k}{m}-\frac{N}{m}\left(1-\frac{1}{p}\right)}, \text { for } 1 \leq p \leq p_{k}^{*}, \quad(k=0,1, \cdots, m-1) ; \\
C(1+t)^{-\gamma}, \text { for } p>p_{k}^{*},
\end{array}\right. \\
& \int_{0}^{\infty}\left\|\partial^{m} u(t)\right\|_{p}^{p} \mathrm{~d} t+\int_{0}^{\infty}\left\|\partial_{t} u(t)\right\|_{p}^{p} \mathrm{~d} t<\infty, \quad \forall p \in(1, m) .
\end{aligned}
$$

Here and hereafter $\partial^{k} u$ represents the vector function $(x, t) \rightarrow\left\{\partial_{x}^{\alpha} u_{j}(x, t):|\alpha|=k, j=\right.$ $1,2, \cdots, n\}$.

Remark. If the function $F$ is locally Hölder continuous, then the solutions to the problem (1.1) obtained by the above theorems are classical ones. This can be proved by following the standard arguement in the theory of parabolic differential equations (c.f. [22,23]).

Remark. If the function $F$ does not involve $\left\{\partial^{\alpha} u: k \leq|\alpha| \leq m-1\right\}(1 \leq k \leq m-1)$, then the condition on the initial function $\varphi$ can be replaced with the weaker condition $\varphi \in C_{B U}^{k-1}\left(R^{N}\right)$ in Theorem 2.1 (resp. $\varphi \in W^{k-1,1}\left(R^{N}\right) \cap C_{B U}^{k-1}\left(R^{N}\right)$ in Theorem 2.2 and 
Theorem 2.3). Of course, the conclusions should be correspondingly modified. See the proofs of these theorems.

Before ending this section we introduce some more notation to be used later. For $s \geq 0,1 \leq p \leq \infty$ and $1 \leq r \leq \infty$, we denote by $B_{p r}^{s}\left(R^{N}\right)$ both the scalar and the $N$ dimensional vector Besov space on $R^{N}$. Recall that for $s=k+\mu\left(k \in Z_{+}^{N}, 0<\mu \leq 1\right)$, $B_{p r}^{s}\left(R^{N}\right)$ is made up of functions $\varphi \in W^{k, p}\left(R^{N}\right)$ satifying $[\varphi]_{s, p, r}<\infty$, where

$$
[\varphi]_{s, p, r}=\left\{\begin{aligned}
& \sum_{|\alpha|=k}\left(\int_{R^{N}}\left(\left\|\partial^{\alpha} \varphi(\cdot+y)-\partial^{\alpha} \varphi\right\|_{p} /|y|^{\mu}\right)^{r} \mathrm{~d} y /|y|^{N}\right)^{\frac{1}{r}} \\
& \text { if } 0<\mu<1,1 \leq r<\infty \\
& \sum_{|\alpha|=k}\left(\int_{R^{N}}\left(\left\|\partial^{\alpha} \varphi(\cdot+y)+\partial^{\alpha} \varphi(\cdot-y)-2 \partial^{\alpha} \varphi\right\|_{p} /|y|\right)^{r} \mathrm{~d} y /|y|^{N}\right)^{\frac{1}{r}} \\
& \text { if } \mu=1,1 \leq r<\infty ; \\
& \lim _{r^{\prime} \rightarrow \infty}[\varphi]_{s, p, r^{\prime}}, \text { if } r=\infty .
\end{aligned}\right.
$$

The norm on $B_{p r}^{s}\left(R^{N}\right)$ is defined by

$$
\|\varphi\|_{s, p, r}=\|\varphi\|_{k, p}+[\varphi]_{s, p, r} .
$$

We will need an equivalent expression of the seminorm $[\cdot]_{s, p, r}$. To introduce it let $\psi$ be a scalar function in $C_{0}^{\infty}\left(R^{N}\right)$ such that

$$
0 \leq \psi \leq 1, \operatorname{supp} \psi \subset\left\{\xi \in R^{N}: 1 / 2 \leq|\xi| \leq 2\right\}, \text { and } \sum_{j=-\infty}^{\infty} \psi\left(2^{-j} \xi\right)=1, \forall \xi \in R^{N} \backslash\{0\},
$$

and let $\varphi_{j}=F^{-1}\left(\psi\left(2^{-j} \cdot\right) \tilde{\varphi}\right)(j=0, \pm 1, \pm 2, \cdots)$, where $F$ and " " represent Fourier transformation. Then we have

$$
\begin{gathered}
{[\varphi]_{s, p, r} \sim\left\{\sum_{j=-\infty}^{\infty}\left(2^{j s}\left\|\varphi_{j}\right\|_{p}\right)^{r}\right\}^{\frac{1}{r}}, \quad \text { if } 1 \leq r<\infty ;} \\
{[\varphi]_{s, p, \infty} \sim \sup _{-\infty<j<\infty}\left(2^{j s}\left\|\varphi_{j}\right\|_{p}\right) .}
\end{gathered}
$$

In the above (and in the following as well) the symbal " " represents equivalence between seminorms. In the following sections we will as usual denote by $[\varphi]_{0, p, r}$ the seminorm obtained from taking $s=0$ at the right sides of the above formulae. Obviously, we have

$$
[\varphi]_{0, p, \infty} \leq\|\varphi\|_{p}
$$

To end this section we point out that since $[\varphi]_{s, p, r_{2}} \leq[\varphi]_{s, p, r_{1}}$ when $r_{1} \leq r_{2}$ (c.f. $[24$, Theorem 6.3.1, p.147]), any bounds for $[\varphi]_{s, p, 1}$ from above are also similar bounds for $[\varphi]_{s, p, r}$ for arbitrary $r \in[1, \infty]$. In the sequel we will use this fact without further explanation. 


\section{Decay and Continuity Modulus Estimates for the Fundamental Solution}

To simplify the notation, from here on we denote by $P\left(\partial_{x}\right)$ the operator $\sum_{|\alpha|=m} A_{\alpha} \partial_{x}^{\alpha}$. Then $\Lambda(\zeta)$ is the maximum of the real parts of all eigenvalues of the matrix $P(i \zeta)$. Let

$$
\tilde{Q}(\zeta, t)=\exp (t P(i \zeta)), \quad \forall \zeta \in C^{N}, \forall t>0 .
$$

Our discussion is started with a basic estimate on this matrix function.

Lemma 3.1 Under the assumption $\left(A_{1}\right)$, there exists a constant $C>0$ depending only on $n$ and $\max _{|\zeta|=1}\|P(i \zeta)\|$ such that

$$
\|\tilde{Q}(\zeta, t)\| \leq C\left(1+t|\zeta|^{m}\right)^{n-1} \mathrm{e}^{-a t|\operatorname{Re} \zeta|^{m}+b t|\operatorname{Im} \zeta|^{m}}, \quad \forall \zeta \in C^{N}, \quad \forall t>0 .
$$

Here (and hereafter) we use the notation $\|\cdot\|$ to denote the norm for matrices.

Proof. By [25, the Lemma following Theorem 1 in Section 6, Chapter 2, Vol.III], we see that for arbitrary $\zeta \in C^{N}$ and $t>0$,

$$
\|\tilde{Q}(\zeta, t)\| \leq\left(1+2 t\|P(i \zeta)\|+\cdots+(2 t\|P(i \zeta)\|)^{n-1}\right) \mathrm{e}^{t \Lambda(\zeta)} .
$$

Therefore, (3.1) follows directly from the assumption $\left(A_{1}\right)$. Q. E. D.

We now define

$$
Q(x, t)=\int_{R^{N}} \mathrm{e}^{i x \xi} \tilde{Q}(\xi, t) \mathrm{d}^{\prime} \xi, \quad \forall x \in R^{N}, \quad \forall t>0,
$$

where $\mathrm{d}^{\prime} \xi=(2 \pi)^{-N} \mathrm{~d} \xi$. By Lemma 3.1, $Q$ is well-defined. It is not other but the fundamental solution of the initial value problem associated to the operator $\partial_{t}-P\left(\partial_{x}\right)$.

Theorem 3.2 Suppose that the assumption $\left(A_{1}\right)$ is satisfied. Let $\alpha \in Z_{+}^{N}, k \in Z_{+}$and $1 \leq \rho \leq \infty$. Then there exist constants $C_{1}>0$ and $C_{2}>0$ depending only on $a, b, m, n$, $\max _{|\zeta|=1}\|P(i \zeta)\|$ and (for $C_{2}$ ) $\rho$ such that for all $t>0$ and $x \in R^{N}$,

$$
\begin{gathered}
\left\|\partial_{t}^{k} \partial_{x}^{\alpha} Q(x, t)\right\| \leq C t^{-\frac{N}{m}-\frac{|\alpha|}{m}-k}\left(1+\left|t^{-\frac{1}{m}} x\right|\right)^{\frac{|\alpha|+m(n+k-1)}{m-1}} \mathrm{e}^{-b^{\prime}\left|t^{-\frac{1}{m}} x\right|^{\frac{m}{m-1}}}, \\
\left\|\partial_{t}^{k}\left(\partial^{\alpha} Q\right)(\cdot, t)\right\|_{\rho} \leq C t^{-\frac{N}{m}\left(1-\frac{1}{\rho}\right)-\frac{|\alpha|}{m}-k},
\end{gathered}
$$

where $b^{\prime}=(m-1) m^{-\frac{1}{m-1}} b^{-\frac{1}{m-1}}$.

Proof. By Cauchy's contour integral theorem, we have

$$
\partial_{t}^{k} \partial_{x}^{\alpha} Q(x, t)=\int_{R^{N}} \mathrm{e}^{i x \xi-x \eta}(i \xi-\eta)^{\alpha} P(i \xi-\eta)^{k} \tilde{Q}(\xi+i \eta, t) \mathrm{d}^{\prime} \xi, \quad \forall x \in R^{N}, \quad \forall t>0,
$$


where $\eta$ is an arbitrary vector in $R^{N}$ independent of the integral variable $\xi$. Let $\nu=|\alpha|+k m$. Then by Lemma 3.1 we have

$$
\begin{aligned}
\left\|\partial_{t}^{k} \partial_{x}^{\alpha} Q(x, t)\right\| & \leq C \mathrm{e}^{-x \eta} \int_{R^{N}}(|\xi|+|\eta|)^{\nu}\|\tilde{Q}(\xi+i \eta, t)\| \mathrm{d}^{\prime} \xi \\
& \leq C \mathrm{e}^{-x \eta+b t|\eta|^{m}} \int_{R^{N}}(|\xi|+|\eta|)^{\nu}\left(1+t|\xi|^{m}+t|\eta|^{m}\right)^{n-1} \mathrm{e}^{-a t|\xi|^{m}} \mathrm{~d}^{\prime} \xi \\
& \leq C \mathrm{e}^{-x \eta+b t|\eta|^{m}} \sum_{\lambda+\mu=\nu} \sum_{p+q=n-1} t^{p}|\eta|^{\lambda+m p} \int_{R^{N}}|\xi|^{\mu}\left(1+t|\xi|^{m}\right)^{q} \mathrm{e}^{-a t|\xi|^{m}} \mathrm{~d}^{\prime} \xi \\
& \leq C t^{-\frac{N}{m}} \mathrm{e}^{-x \eta+b t|\eta|^{m}} \sum_{\lambda+\mu=\nu} \sum_{p=0}^{n-1} t^{p-\frac{\mu}{m}}|\eta|^{\lambda+m p}
\end{aligned}
$$

Now for $x \neq 0$ take

$$
\eta=(m b)^{-\frac{1}{m-1}} t^{-\frac{1}{m-1}}|x|^{\frac{2-m}{m-1}} x
$$

and put it into (3.4). Then we obtain (3.2). (3.3) follows directly from (3.2). Q. E. D.

Now for $t>0$ we denote by $S(t)$ the mapping from $S^{\prime}\left(R^{N}\right)$ to $C^{\infty}\left(R^{N}\right)$ defined as follows:

$$
S(t): \varphi \rightarrow S(t) \varphi=Q(\cdot, t) * \varphi, \quad \forall \varphi \in S^{\prime}\left(R^{N}\right),
$$

where "*" represents convolution. By applying Theorem 3.2 and Young's inequality for convolution, we get

Theorem 3.3 Suppose that the assumption $\left(A_{1}\right)$ is satisfied. Let $1 \leq p \leq \infty$ and suppose that $\varphi \in L^{p}\left(R^{N}\right)$. Then $S(\cdot) \varphi \in C^{\infty}\left((0, \infty) ; W^{\infty, p}\left(R^{N}\right) \cap W^{\infty, \infty}\left(R^{N}\right)\right)$ and for any $k \in Z_{+}, \alpha \in Z_{+}^{N}$ and $q \in[p, \infty]$ there exists a corresponding constant $C>0$ such that

$$
\left\|\partial_{t}^{k} \partial^{\alpha} S(t) \varphi\right\|_{q} \leq C t^{-\frac{N}{m}\left(\frac{1}{p}-\frac{1}{q}\right)-\frac{|\alpha|}{m}-k}\|\varphi\|_{p}, \quad \forall t>0 .
$$

If furthermore $\varphi \in W^{k, p}\left(R^{N}\right)\left(k \in Z_{+}, 1 \leq p \leq \infty\right)$, then we have

$$
\left\|\partial_{t}^{k} \partial^{\alpha} S(t) \varphi\right\|_{q} \leq C t^{-\frac{N}{m}\left(\frac{1}{p}-\frac{1}{q}\right)-k}\left\|\partial^{\alpha} \varphi\right\|_{p}, \quad \forall t>0, \quad \forall|\alpha| \leq k, \quad \forall q \in[p, \infty] .
$$

In particular,

$$
\begin{aligned}
& \left\|\partial^{\alpha} S(t) \varphi\right\|_{p} \leq C t^{-\frac{|\alpha|}{m}}\|\varphi\|_{p}, \quad \forall t>0 ; \\
& \left\|\partial^{\alpha} S(t) \varphi\right\|_{p} \leq C\left\|\partial^{\alpha} \varphi\right\|_{p}, \quad \forall t>0 ;
\end{aligned}
$$

whenever the right side makes sense. Q. E. D.

Corollary 3.4 Suppose that the assumption $\left(A_{1}\right)$ is satisfied and $1 \leq p \leq q \leq \infty$. Then

$$
\begin{array}{cc}
\left\|\partial^{\alpha} S(t) \varphi\right\|_{q} \leq C t^{-\frac{|\alpha|}{m}}(1+t)^{-\frac{N}{m}\left(\frac{1}{p}-\frac{1}{q}\right)}\left(\|\varphi\|_{p}+\|\varphi\|_{q}\right), \quad \forall t>0 \\
\left\|\partial^{\alpha} S(t) \varphi\right\|_{q} \leq C(1+t)^{-\frac{N}{m}\left(\frac{1}{p}-\frac{1}{q}\right)-\frac{|\alpha|}{m}}\left(\|\varphi\|_{p}+\left\|\partial^{\alpha} \varphi\right\|_{q}\right), \quad \forall t>0
\end{array}
$$


whenever the right side makes sense. Q. E. D.

Theorem 3.5 Suppose that the assumption $\left(A_{1}\right)$ is satisfied. Let $k \in Z_{+}, \alpha \in$ $Z_{+}^{N}, r>0, s \geq 0$ and $|\alpha|+k m+r>s$. Let $1 \leq p \leq q \leq \infty$. Then there exists a constant $C>0$ such that

$$
\left[\partial_{t}^{k} \partial^{\alpha} S(t) \varphi\right]_{r, q, 1} \leq C t^{-\frac{N}{m}\left(\frac{1}{p}-\frac{1}{q}\right)-\frac{|\alpha|+r-s}{m}-k}[\varphi]_{s, p, \infty}, \quad \forall t>0,
$$

whenever the right side makes sense.

Proof. Since $\partial_{t}^{k} S(t) \varphi=P(\partial)^{k} S(t) \varphi$, we see that

$$
\left[\partial_{t}^{k} \partial^{\alpha} S(t) \varphi\right]_{r, q, 1} \leq C[S(t) \varphi]_{r+|\alpha|+k m, q, 1} .
$$

Thus we only need to consider the case where $\alpha=0$ and $k=0$. Let $\psi$ be as in the above section, and let $\varphi \sim \sum_{j=-\infty}^{\infty} \varphi_{j}$ be the corresponding homogeneous Littlewood-Paley decomposition of $\varphi$, i.e., $\tilde{\varphi_{j}}(\xi)=\psi\left(2^{-j} \xi\right) \tilde{\varphi}(\xi)(j=0, \pm 1, \pm 2, \cdots)$. Then, as one can easily verify, the corresponding decomposition of $S(t) \varphi$ is $\sum_{j=-\infty}^{\infty} \omega_{j}(\cdot, t)$, where

$$
\tilde{\omega}_{j}(\xi, t)=\tilde{Q}(\xi, t) \tilde{\varphi_{j}}(\xi), \quad j=0, \pm 1, \pm 2, \cdots .
$$

Take a scalar function $\chi \in C_{0}^{\infty}\left(R^{N}\right)$ such that

$$
\operatorname{supp} \chi \subset\left\{\xi \in R^{N}: 1 / 4 \leq|\xi| \leq 4\right\} ; \quad \chi(\xi)=1 \text { for } 1 / 2 \leq|\xi| \leq 2 ;
$$

and let $A_{j}(\xi, t)=\chi\left(2^{-j} \xi\right) \tilde{Q}(\xi, t)(j=0, \pm 1, \pm 2, \cdots)$. Then $\tilde{\omega}_{j}(\xi, t)=A_{j}(\xi, t) \tilde{\varphi_{j}}(\xi)(j=$ $0, \pm 1, \pm 2, \cdots)$ because $\chi\left(2^{-j} \xi\right) \tilde{\varphi_{j}}(\xi)=\tilde{\varphi}_{j}(\xi)$. Therefore, we have

$$
[S(t) \varphi]_{r, q, 1}=\sum_{j=-\infty}^{\infty} 2^{j r}\left\|\omega_{j}(\cdot, t)\right\|_{q} \leq \sum_{j=-\infty}^{\infty} 2^{j r} M_{q}\left(A_{j}(\cdot, t)\right)\left\|\varphi_{j}\right\|_{q}
$$

where $M_{q}\left(A_{j}(\cdot, t)\right)$ is the modulus of $A_{j}(\cdot, t)$ as Fourier multiplier on $L^{q}\left(R^{N}\right)$. By virtue of Carlson-Beurling's inequality (c.f. [24, Lemma 6.1.5, p.135]) we have, for arbitrary integer $L>N / 2$,

$$
M_{q}\left(A_{j}(\cdot, t)\right) \leq C\left\|A_{j}(\cdot, t)\right\|_{2}^{1-\frac{N}{2 L}}\left(\sup _{|\beta|=L}\left\|\partial^{\beta} A_{j}(\cdot, t)\right\|_{2}\right)^{\frac{N}{2 L}},
$$

where $C$ is a positive constant depending only on $n, N$ and $L$ (hence, it is independent of $j$ ). Since $A_{j}(\xi, t)$ is identically zero outside the region $2^{j-2} \leq|\xi| \leq 2^{j+2}$, by applying Lemma 3.1 we get

$$
\begin{gathered}
\left\|A_{j}(\xi, t)\right\| \leq C \mathrm{e}^{-\frac{1}{2} a t|\xi|^{m}} \leq C \mathrm{e}^{-c t 2^{j m}} \\
\left\|\partial^{\beta} A_{j}(\xi, t)\right\| \leq C \sum_{|\beta| / m \leq l \leq|\beta|}|\xi|^{m l-|\beta|} t^{l} \mathrm{e}^{-\frac{1}{2} a t|\xi|^{m}} \leq C t^{\frac{|\beta|}{m}} \mathrm{e}^{-c t 2^{j m}},
\end{gathered}
$$

where $c=4^{-(m+1)} a$. Hence, due to the same reason, we have

$$
\left\|A_{j}(\cdot, t)\right\|_{2} \leq C 2^{\frac{j N}{2}} \mathrm{e}^{-c t 2^{j m}}, \quad\left\|\partial^{\beta} A_{j}(\cdot, t)\right\|_{2} \leq C 2^{\frac{j N}{2}} t^{\frac{|\beta|}{m}} \mathrm{e}^{-c t 2^{j m}} .
$$


Putting these estimates into (3.13), we obtain

$$
M_{q}\left(A_{j}(\cdot, t)\right) \leq C 2^{\frac{j N}{2}} t^{\frac{N}{2 m}} \mathrm{e}^{-c t 2^{j m}}, \forall t>0, j=0, \pm 1, \pm 2, \cdots .
$$

Similarly, by writing $\varphi_{j}=F^{-1}\left(\chi\left(2^{-j} \cdot\right) \varphi_{j}\right)$, we get

$$
\left\|\varphi_{j}\right\|_{q} \leq C 2^{j N\left(\frac{1}{p}-\frac{1}{q}\right)}\left\|\varphi_{j}\right\|_{p}, \quad \forall t>0, \quad j=0, \pm 1, \pm 2, \cdots .
$$

Combining (3.12), (3.14) and (3.15) together, we get

$$
\begin{aligned}
{[S(t) \varphi]_{r, q, 1} } & \leq C t^{\frac{N}{2 m}} \sum_{j=-\infty}^{\infty} 2^{j\left(r+N\left(\frac{1}{p}-\frac{1}{q}\right)+\frac{N}{2}\right)} \mathrm{e}^{-c t 2^{j m}}\left\|\varphi_{j}\right\|_{p} \\
& \leq C t^{\frac{N}{2 m}} \sum_{j=-\infty}^{\infty} 2^{j\left(r-s+N\left(\frac{1}{p}-\frac{1}{q}\right)+\frac{N}{2}\right)} \mathrm{e}^{-c t 2^{j m}} \cdot \sup _{j}\left(2^{j s}\left\|\varphi_{j}\right\|_{p}\right) \\
& \leq C t^{\frac{N}{2 m}} \sum_{j=-\infty}^{\infty} \int_{2^{j-1}}^{2^{j}} \sigma^{r-s+N\left(\frac{1}{p}-\frac{1}{q}\right)+\frac{N}{2}} \mathrm{e}^{-c t \sigma^{m}} \mathrm{~d} \sigma \cdot[\varphi]_{s, p, \infty} \\
& \leq C t^{-\frac{r-s}{m}-\frac{N}{m}\left(\frac{1}{p}-\frac{1}{q}\right)}[\varphi]_{s, p, \infty} .
\end{aligned}
$$

This finishes the proof. Q. E. D.

By making application of the above theorem and (2.9), we get

Corollary 3.6 Let $1 \leq p \leq q \leq \infty, 0<\theta<1$ and $\alpha \in Z_{+}^{N}$. Then under the assumption $\left(A_{1}\right)$ we have

$$
\begin{gathered}
{\left[\partial^{\alpha} S(t) \varphi\right]_{\theta, q, 1} \leq C t^{-\frac{|\alpha|+\theta}{m}}(1+t)^{-\frac{N}{m}\left(\frac{1}{p}-\frac{1}{q}\right)}\left(\|\varphi\|_{p}+\|\varphi\|_{q}\right), \quad \forall t>0,} \\
{\left[\partial^{\alpha} S(t) \varphi\right]_{\theta, q, 1} \leq C t^{-\frac{\theta}{m}}(1+t)^{-\frac{|\alpha|}{m}-\frac{N}{m}\left(\frac{1}{p}-\frac{1}{q}\right)}\left(\|\varphi\|_{p}+\left\|\partial^{\alpha} \varphi\right\|_{q}\right), \quad \forall t>0,}
\end{gathered}
$$

whenever the right side makes sense. Q. E. D.

Hereafter we let $S(0) \varphi=\varphi$.

Theorem 3.7 (i) Suppose that the assumption $\left(A_{1}\right)$ is satisfied. Let $1 \leq p<\infty$ and suppose that $\varphi \in L^{p}\left(R^{N}\right)$. Then $S(\cdot) \varphi \in C\left([0, \infty) ; L^{p}\left(R^{N}\right)\right) \cap C\left((0, \infty) ; W^{\infty, p}\left(R^{N}\right)\right)$. Moreover, for arbitrary $\alpha \in Z_{+}$and $s>0$ there exist corresponding constants $C_{1}=C_{1}(\alpha, p)>0$ and $C_{2}=C_{2}(s, p)>0$ such that

$$
\begin{gathered}
\left\|\partial^{\alpha} S(t) \varphi-\partial^{\alpha} S\left(t^{\prime}\right) \varphi\right\|_{p} \leq C_{1}\left(\min \left(t, t^{\prime}\right)\right)^{-1-\frac{|\alpha|}{m}}\left|t-t^{\prime}\right|\|\varphi\|_{p}, \quad \forall t, t^{\prime} \in(0 ; \infty) \\
{\left[S(t) \varphi-S\left(t^{\prime}\right) \varphi\right]_{s, p, 1} \leq C_{2}\left(\min \left(t, t^{\prime}\right)\right)^{-1-\frac{s}{m}} \mid t-t^{\prime}\|\varphi\|_{p}, \quad \forall t, t^{\prime} \in(0, \infty) .}
\end{gathered}
$$

If furthermore $\varphi \in W^{m-1 . p}\left(R^{N}\right)$, then $S(\cdot) \varphi \in C\left([0, \infty) ; W^{m-1, p}\left(R^{N}\right)\right) \cap C((0, \infty)$; $\left.W^{\infty, p}\left(R^{N}\right)\right)$. Moreover, for any $\alpha \in Z_{+}(|\alpha| \leq m-2)$ and $s \in(0, m-1)$ there exist corresponding constants $C_{1}=C_{1}(\alpha, p)>0$ and $C_{2}=C_{2}(s, p)>0$ such that

$$
\begin{gathered}
\left\|\partial^{\alpha} S(t) \varphi-\partial^{\alpha} S\left(t^{\prime}\right) \varphi\right\|_{p} \leq C_{1}\left|t-t^{\prime}\right|^{1-\frac{|\alpha|+1}{m}}\|\varphi\|_{m-1, p}, \quad \forall t, t^{\prime} \in(0 ; \infty) ; \\
{\left[S(t) \varphi-S\left(t^{\prime}\right) \varphi\right]_{s, p, 1} \leq C_{2}\left|t-t^{\prime}\right|^{1-\frac{s+1}{m}}\|\varphi\|_{m-1, p}, \quad \forall t, t^{\prime} \in(0, \infty) .}
\end{gathered}
$$


(ii) Suppose that the assumption $\left(A_{1}\right)$ is satisfied and $\varphi \in C_{B U}\left(R^{N}\right)$. Then $S(\cdot) \varphi \in$ $C\left([0, \infty) ; C_{B U}\left(R^{N}\right)\right) \cap C\left((0, \infty) ; W^{\infty, \infty}\left(R^{N}\right)\right)$. Moreover, (3.18) and (3.19) are valid with $p$ replaced with $\infty$. If furthermore $\varphi \in C_{B U}^{m-1}\left(R^{N}\right)$, then $S(\cdot) \varphi \in C\left([0, \infty) ; C_{B U}^{m-1}\left(R^{N}\right)\right) \cap$ $C\left((0, \infty) ; W^{\infty, \infty}\left(R^{N}\right)\right)$. Moreover, for any $\alpha \in Z_{+}(|\alpha| \leq m-2)$ and $s \in(0, m-1)$ there exist corresponding constants $C_{1}=C_{1}(\alpha, p)>0$ and $C_{2}=C_{2}(s, p)>0$ such that (3.20) and (3.21) are valid with $p$ replaced by $\infty$.

Proof. The assertion that $S(\cdot) \varphi \in C\left([0, \infty) ; L^{p}\left(R^{N}\right)\right)$ (resp. $S(\cdot) \varphi \in C([0, \infty)$; $\left.W^{m-1, p}\left(R^{N}\right)\right)$ ) when $\varphi \in L^{p}\left(R^{N}\right)$ (resp. when $\left.\varphi \in W^{m-1, p}\left(R^{N}\right)\right)$ follows from (3.18) and the fact that $\lim _{t \rightarrow 0+} S(t) \varphi=\varphi$ in $L^{p}\left(R^{N}\right)$ (resp. in $W^{m-1, p}\left(R^{N}\right)$ ), of which the later can be proved by using the standard method for similar arguements in potential theory (c.f. the proof of [26, Theorem 1 and 2, Chapter III, p.62-64]) and is hence omitted here. To prove (3.18), without loss of generality we may assume $t^{\prime}>t$. Then by virtue of Theorem 3.3 we have

$$
\begin{aligned}
\left\|\partial^{\alpha} S(t) \varphi-\partial^{\alpha} S\left(t^{\prime}\right) \varphi\right\|_{p} & =\left\|\int_{t}^{t^{\prime}} \partial_{\tau} \partial^{\alpha} S(\tau) \varphi \mathrm{d} \tau\right\|_{p} \leq \int_{t}^{t^{\prime}}\left\|\partial_{\tau} \partial^{\alpha} S(\tau) \varphi\right\|_{p} \mathrm{~d} \tau \\
& \leq C \int_{t}^{t^{\prime}} \tau^{-1-\frac{|\alpha|}{m}} \mathrm{~d} \tau \cdot\|\varphi\|_{p} \leq C t^{-1-\frac{|\alpha|}{m}}\left(t^{\prime}-t\right)\|\varphi\|_{p}
\end{aligned}
$$

which proves (3.18). The proof of (3.19) is similar. To prove (3.20), we assume that $t^{\prime}>t$ and write

$$
\begin{aligned}
\partial^{\alpha} S\left(t^{\prime}\right) \varphi-\partial^{\alpha} S(t) \varphi & =\int_{t}^{t^{\prime}} \partial_{\tau} \partial^{\alpha} S(\tau) \varphi \mathrm{d} \tau=\int_{t}^{t^{\prime}} P(\partial) \partial^{\alpha} S(\tau) \varphi \mathrm{d} \tau \\
& =\sum_{|\beta|=|\alpha|+1} \sum_{\left|\alpha^{\prime}\right|=m-1} C_{\beta \alpha^{\prime}} \int_{t}^{t^{\prime}} \partial^{\beta} S(\tau) \partial^{\alpha^{\prime}} \varphi \mathrm{d} \tau,
\end{aligned}
$$

where $C_{\beta \alpha^{\prime}}$ 's are constant matrices. If $|\alpha| \leq m-2$ then $|\beta| \leq m-1$. Thus by (3.7), Theorem 3.3 , we get

$$
\begin{aligned}
\left\|\partial^{\alpha} S\left(t^{\prime}\right) \varphi-\partial^{\alpha} S(t) \varphi\right\|_{p} & \leq C \sum_{|\beta|=|\alpha|+1} \sum_{\left|\alpha^{\prime}\right|=m-1} \int_{t}^{t^{\prime}} \tau^{-\frac{|\beta|}{m}}\left\|\partial^{\alpha^{\prime}} \varphi\right\|_{p} \mathrm{~d} \tau \\
& \leq C\left(t^{\prime}-t\right)^{1-\frac{|\alpha|+1}{m}}\|\varphi\|_{m-1, p} .
\end{aligned}
$$

This proves (3.20). The proof of (3.21) is similar. The conclusion (ii) follows from a similar procedure as above, thus its proof is omitted. Q. E. D.

Theorem 3.8 Suppose that the assumption $\left(A_{1}\right)$ is satisfied. Suppose that $1 \leq p<\infty$ and $\varphi \in B_{p p}^{m\left(1-\frac{1}{p}\right)}\left(R^{N}\right)$ (if $m(1-1 / p)$ is not an integer) or $\varphi \in W^{k, p}\left(R^{N}\right)$ (if $m(1-1 / p)=$ $\left.k \in Z_{+}\right)$. Then $S(\cdot) \varphi \in L_{\text {loc }}^{p}\left([0, \infty) ; W^{m, p}\left(R^{N}\right)\right) \cap W^{1, p}\left([0, \infty) ; L^{p}\left(R^{N}\right)\right)$. Moreover, there exists a constant $C>0$ independent of $\varphi$ such that

$$
\begin{gathered}
\int_{0}^{\infty}\left\|\partial_{t} S(t) \varphi\right\|_{p}^{p} \mathrm{~d} t+\sum_{|\alpha|=m} \int_{0}^{\infty}\left\|\partial^{\alpha} S(t) \varphi\right\|_{p}^{p} \mathrm{~d} t \leq C[\varphi]_{m\left(1-\frac{1}{p}\right), p, p}^{p}, \text { if } m\left(1-\frac{1}{p}\right) \notin Z_{+} ; \quad \text { (3.22) } \\
\int_{0}^{T}\left\|\partial_{t} S(t) \varphi\right\|_{p}^{p} \mathrm{~d} t+\sum_{|\alpha|=m} \int_{0}^{T}\left\|\partial^{\alpha} S(t) \varphi\right\|_{p}^{p} \mathrm{~d} t \leq C T^{\frac{k}{m}}\|\varphi\|_{k, p}^{p}, \quad \forall T>0, \text { if } m\left(1-\frac{1}{p}\right)=k \in Z_{+} .
\end{gathered}
$$


Proof. Let $w(\cdot, t)=S(t) \varphi(\cdot)=Q(\cdot, t) * \varphi(\cdot)$. We first assume that $m(1-1 / p) \notin Z_{+}$. Denote by $k$ the largest integer smaller than $m(1-1 / p)$. Then since $\int_{R^{N}} \partial_{x}^{\beta} Q(x, t) \mathrm{d} x=0$ for $\beta \neq 0$, we have

$$
\begin{aligned}
\partial_{t} w(x, t) & =\int_{R^{N}} \partial_{t} Q(y, t) \varphi(x-y) \mathrm{d} y=\int_{R^{N}} P\left(\partial_{y}\right) Q(y, t) \varphi(x-y) \mathrm{d} y \\
& =\sum_{|\beta|=m-k} \sum_{|\alpha|=k} C_{\alpha \beta} \int_{R^{N}} \partial_{y}^{\beta} Q(y, t) \partial_{x}^{\alpha} \varphi(x-y) \mathrm{d} y \\
& =\sum_{|\beta|=m-k} \sum_{|\alpha|=k} C_{\alpha \beta} \int_{R^{N}} \partial_{y}^{\beta} Q(y, t)\left(\partial_{x}^{\alpha} \varphi(x-y)-\partial_{x}^{\alpha} \varphi(x)\right) \mathrm{d} y,
\end{aligned}
$$

where $C_{\alpha \beta}$ 's are constant matrices. From the above equality, by making application of Hölder's inequality we obtain

$$
\begin{gathered}
\left\|\partial_{t} w(\cdot, t)\right\|_{p} \leq C \sum_{|\beta|=m-k} \sum_{|\alpha|=k} \int_{R^{N}}\left\|\partial_{y}^{\beta} Q(y, t)\right\|\left\|\partial^{\alpha} \varphi(\cdot-y)-\partial^{\alpha} \varphi\right\|_{p} \mathrm{~d} y \\
\leq C \sum_{|\beta|=m-k} \sum_{|\alpha|=k}\left(\int_{R^{N}}\left\|\partial_{y}^{\beta} Q(y, t)\right\| \mathrm{d} y\right)^{1-\frac{1}{p}} \\
\cdot\left(\int_{R^{N}}\left\|\partial_{y}^{\beta} Q(y, t)\right\|\left\|\partial^{\alpha} \varphi(\cdot-y)-\partial^{\alpha} \varphi\right\|_{p}^{p} \mathrm{~d} y\right)^{\frac{1}{p}} \cdot
\end{gathered}
$$

Applying (3.3) of Theorem 3.2 we get

$$
\int_{R^{N}}\left\|\partial_{y}^{\beta} Q(y, t)\right\| \mathrm{d} y \leq C t^{-\frac{|\beta|}{m}}, \forall t>0
$$

Therefore,

$$
\left\|\partial_{t} w(\cdot, t)\right\|_{p} \leq C \sum_{|\beta|=m-k} \sum_{|\alpha|=k} t^{-\frac{|\beta|}{m}\left(1-\frac{1}{p}\right)}\left(\int_{R^{N}}\left\|\partial_{y}^{\beta} Q(y, t)\right\|\left\|\partial^{\alpha} \varphi(\cdot-y)-\partial^{\alpha} \varphi\right\|_{p^{p}}^{p} y\right)^{\frac{1}{p}} .
$$

Consequently,

$$
\int_{0}^{\infty}\left\|\partial_{t} w(\cdot, t)\right\|_{p}^{p} \mathrm{~d} t \leq C \sum_{|\beta|=m-k} \sum_{|\alpha|=k} \int_{R^{N}}\left(\int_{0}^{\infty} t^{-\frac{|\beta|}{m}(p-1)}\left\|\partial_{y}^{\beta} Q(y, t)\right\| \mathrm{d} t\right)\left\|\partial^{\alpha} \varphi(\cdot-y)-\partial^{\alpha} \varphi\right\|_{p}^{p} \mathrm{~d} y .
$$

Applying (3.2) of Theorem 3.2 we get for $|\beta|=m-k$ and $y \neq 0$,

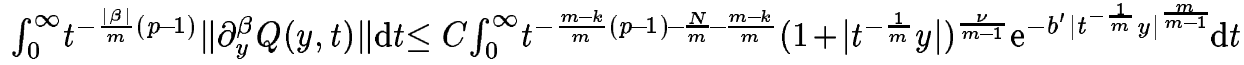

$$
\begin{aligned}
& \leq C|y|^{-p\left(m\left(1-\frac{1}{p}\right)-k\right)-N},
\end{aligned}
$$

where $\nu=m-k+m(n-1)$. We note that the integrals in the above inequalities make sense because $\frac{m-k}{m}(p-1)+\frac{N}{m}+\frac{m-k}{m}=\frac{m-k}{m} p+\frac{N}{m}>1$ by the hypothesis $m\left(1-\frac{1}{p}\right)>k$, and 
$\lim _{t \rightarrow 0^{+}} t^{-a} \mathrm{e}^{-c t^{-b}}=0$ for arbitrary positive $a, b$ and $c$. Hence

$$
\int_{0}^{\infty}\left\|\partial_{t} w(\cdot, t)\right\|_{p}^{p} \mathrm{~d} t \leq C \sum_{|\alpha|=k} \int_{R^{N}}|y|^{-p\left(m\left(1-\frac{1}{p}\right)-k\right)-N}\left\|\partial^{\alpha} \varphi(\cdot-y)-\partial^{\alpha} \varphi\right\|_{p}^{p} \mathrm{~d} y=C[\varphi]_{m\left(1-\frac{1}{p}\right), p, p}^{p}
$$

Similarly we can obtain

$$
\sum_{|\alpha|=m} \int_{0}^{\infty}\left\|\partial_{x}^{\alpha} w(\cdot, t)\right\|_{p}^{p} \mathrm{~d} t \leq C[\varphi]_{m\left(1-\frac{1}{p}\right), p, p}^{p} .
$$

Combining (3.26) and (3.27) together we get (3.22). The proof of (3.23) is similar and easier. We omit it here. Q. E. D.

\section{Decay and Continuity Modulus Estimates for the Potential Integral}

In this section we make estimates to the following potential integral:

$$
w(t)=\int_{0}^{t} S(t-\tau) F(\tau) \mathrm{d} \tau(t>0), \quad w(0)=0,
$$

where $F(t)=F(\cdot, t)$ is a suitable measurable function. We need the following preliminary lemma:

Lemma 4.1 Suppose that $0 \leq \theta<1, a \geq 0$ and $b \geq 0$. Then there exists constant $C>0$ depending only on $a, b$ and $\theta$ such that for all $t>0$,

$$
\int_{0}^{t}(t-\tau)^{-\theta}(1+t-\tau)^{-a}(1+\tau)^{-b} \mathrm{~d} \tau \leq \begin{cases}C(1+t)^{-\min (a+\theta, b)}, & \text { if } \max (a+\theta, b)>1, \\ C(1+t)^{-\min (a+\theta, b)} \ln (2+t), & \text { if } \max (a+\theta, b)=1, \\ C(1+t)^{1-a-\theta-b}, & \text { if } \max (a+\theta, b)<1 .\end{cases}
$$

Proof. First we assume that $t \leq 2$. Then

$$
\int_{0}^{t}(t-\tau)^{-\theta}(1+t-\tau)^{-a}(1+\tau)^{-b} \mathrm{~d} \tau \leq \int_{0}^{t}(t-\tau)^{-\theta} \mathrm{d} \tau=\frac{t^{1-\theta}}{1-\theta} \leq \frac{2^{1-\theta}}{1-\theta} .
$$

Next we assume $t \geq 2$. Then

$$
\int_{0}^{t}(t-\tau)^{-\theta}(1+t-\tau)^{-a}(1+\tau)^{-b} \mathrm{~d} \tau=\int_{0}^{\frac{t}{2}}+\int_{\frac{t}{2}}^{t} .
$$


For the first integral on the right hand side, we have

$$
\int_{0}^{\frac{t}{2}}(t-\tau)^{-\theta}(1+t-\tau)^{-a}(1+\tau)^{-b} \mathrm{~d} \tau \leq \begin{cases}\left(\frac{t}{2}\right)^{-(a+\theta)} \int_{0}^{\infty}(1+\tau)^{-b} \mathrm{~d} \tau, & \text { if } b>1, \\ \left(\frac{t}{2}\right)^{-(a+\theta)} \ln \left(1+\frac{t}{2}\right), & \text { if } b=1, \\ \frac{1}{1-b}\left(\frac{t}{2}\right)^{-(a+\theta)}\left(1+\frac{t}{2}\right)^{1-b}, & \text { if } b<1 .\end{cases}
$$

For the second integral, we have

$$
\begin{aligned}
& \int_{\frac{t}{2}}^{t}(t-\tau)^{-\theta}(1+t-\tau)^{-a}(1+\tau)^{-b} \mathrm{~d} \tau \leq\left(1+\frac{t}{2}\right)^{-b} \int_{\frac{t}{2}}^{t}(t-\tau)^{-\theta}(1+t-\tau)^{-a} \mathrm{~d} \tau \\
= & \frac{1}{1-\theta}\left(\frac{t}{2}\right)^{1-\theta}\left(1+\frac{t}{2}\right)^{-a-b}+\frac{a}{1-\theta}\left(1+\frac{t}{2}\right)^{-b} \int_{0}^{\frac{t}{2}} \tau^{1-\theta}(1+\tau)^{-a-1} \mathrm{~d} \tau \\
\leq & \frac{1}{1-\theta}\left(\frac{t}{2}\right)^{1-\theta}\left(1+\frac{t}{2}\right)^{-a-b}+\frac{a}{1-\theta}\left(1+\frac{t}{2}\right)^{-b} \int_{0}^{\frac{t}{2}}(1+\tau)^{-a-\theta} \mathrm{d} \tau \\
\leq & \begin{cases}\frac{1}{1-\theta}\left(\frac{t}{2}\right)^{1-\theta-a-b}+\frac{a}{1-\theta}\left(\frac{t}{2}\right)^{-b} \int_{0}^{\infty}(1+\tau)^{-a-\theta} \mathrm{d} \tau, & \text { if } a+\theta>1, \\
\frac{1}{1-\theta}\left(\frac{t}{2}\right)^{1-\theta-a-b}+\frac{a}{1-\theta}\left(\frac{t}{2}\right)^{-b} \ln \left(1+\frac{t}{2}\right), & \text { if } a+\theta=1, \\
\frac{1}{1-\theta}\left(\frac{t}{2}\right)^{1-\theta-a-b}+\frac{a}{(1-\theta)(1-\theta-a)}\left(1+\frac{t}{2}\right)^{1-\theta-a-b}, & \text { if } a+\theta<1 .\end{cases}
\end{aligned}
$$

Now, (4.2) follows easily from (4.3)-(4.5). Q. E. D.

Theorem 4.2 Suppose that the operator $\partial_{t}-P\left(\partial_{x}\right)$ satisfies the assumption $\left(A_{1}\right)$. Suppose that $1 \leq p \leq q \leq \infty$ and $0<T \leq \infty$. Suppose furthermore that $F \in L^{\infty}([0, T)$; $\left.L^{p}\left(R^{N}\right) \cap L^{q}\left(R^{\bar{N}}\right)\right)$ and there exist constants $\kappa \geq 0$ and $M>0$ such that

$$
\|F(t)\|_{p}+\|F(t)\|_{q} \leq M(1+t)^{-\kappa}, \quad \forall t \in[0, T) .
$$

Then for any $0<\theta<1$ the function $w$ given in (4.1) belongs to $L^{\infty}\left([0, T) ; B_{p 1}^{m-1+\theta}\left(R^{N}\right) \cap\right.$ $\left.B_{q 1}^{m-1+\theta}\left(R^{N}\right)\right)$. Moreover, for any $0 \leq k \leq m-1\left(k \in Z_{+}\right)$and $0<\theta<1$, there exist corresponding constants $C_{1}=C_{1}(k, p, q)>0$ and $C_{2}=C_{2}(\theta, p, q)>0$ independent of $T, M$ and $F$ such that for all $t \in[0, T)$,

$$
\left\|\partial^{k} w(t)\right\|_{q} \leq\left\{\begin{array}{c}
C_{1} M(1+t)^{-} \min \left(\frac{k}{m}+\frac{N}{m}\left(\frac{1}{p}-\frac{1}{q}\right), \kappa\right) \\
\text { if } \max \left(\frac{k}{m}+\frac{N}{m}\left(\frac{1}{p}-\frac{1}{q}\right), \kappa\right)>1, \\
C_{1} M(1+t)^{-\min \left(\frac{k}{m}+\frac{N}{m}\left(\frac{1}{p}-\frac{1}{q}\right), \kappa\right)} \ln (2+t), \\
\text { if } \max \left(\frac{k}{m}+\frac{N}{m}\left(\frac{1}{p}-\frac{1}{q}\right), \kappa\right)=1, \\
C_{1} M(1+t)^{1-\frac{k}{m}-\frac{N}{m}\left(\frac{1}{p}-\frac{1}{q}\right)-\kappa}, \\
\text { if } \max \left(\frac{k}{m}+\frac{N}{m}\left(\frac{1}{p}-\frac{1}{q}\right), \kappa\right)<1,
\end{array}\right.
$$




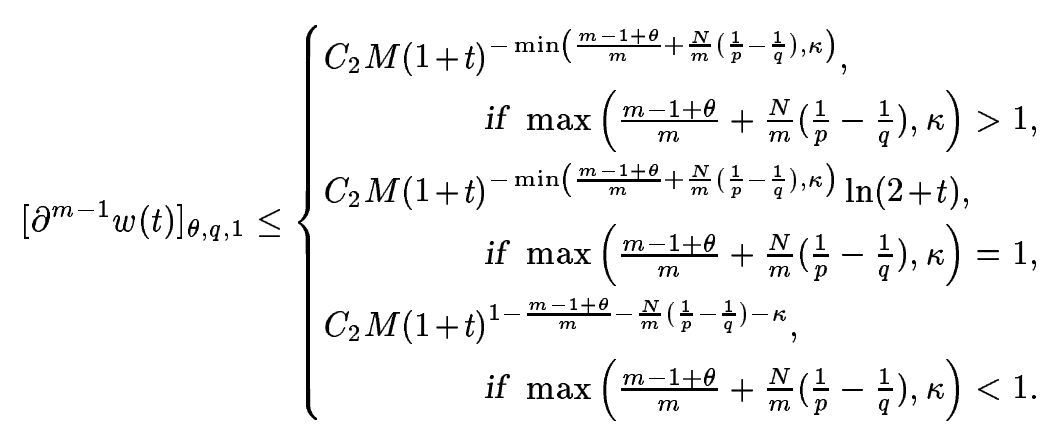

Proof. Since $0 \leq k \leq m-1$, by (3.9), Corollary 3.5, we have

$$
\left\|\partial^{k} w(t)\right\|_{q} \leq C \int_{0}^{t}(t-\tau)^{-\frac{k}{m}}(1+t-\tau)^{-\frac{N}{m}\left(\frac{1}{p}-\frac{1}{q}\right)}\left(\|F(\tau)\|_{p}+\|F(\tau)\|_{q}\right) \mathrm{d} \tau,
$$

where $C$ is a positive constant depending only on $k, p, q, m, n, N$ and $\max _{|\zeta|=1}\|P(i \zeta)\|$. From this estimate, by making application of Lemma 4.1, we immediately get (4.7). Similarly, since $m-1+\theta<m$, by making application of (3.16), Corollary 3.6, and Lemma 4.1 once again, we get (4.8). Q. E. D.

Theorem 4.3 (i) Let $1 \leq p<\infty$ and $0<T \leq \infty$. Suppose that $F \in L^{\infty}\left([0, T) ; L^{p}\left(R^{N}\right)\right)$ and there is a constant $M>0$ such that $\|F(t)\|_{p} \leq M$ for all $t \in[0, T)$. Then for any $0<$ $\theta<1$ the function $w$ given in (4.1) belongs to $C\left([0, T) ; B_{p 1}^{m-1+\theta}\left(R^{N}\right)\right) \cap C^{1 \#}\left([0, T) ; L^{p}\left(R^{N}\right)\right)$. Moreover, for any $0 \leq k \leq m-1\left(k \in Z_{+}\right)$and any $0<\theta<1$ there exist corresponding constants $C_{1}=C_{1}(k, p)>0$ and $C_{2}=C_{2}(\theta, p)>0$ independent of $T, M$ and $F$ such that

$$
\begin{gathered}
\left\|w(t)-w\left(t^{\prime}\right)\right\|_{p} \leq-C_{1} M\left|t-t^{\prime}\right| \ln \left|t-t^{\prime}\right|, \quad \forall t, t^{\prime} \in(0, T)\left(0<\left|t-t^{\prime}\right| \leq \frac{1}{\mathrm{e}}\right) ; \\
\left\|\partial^{k} w(t)-\partial^{k} w\left(t^{\prime}\right)\right\|_{p} \leq C_{1} M\left|t-t^{\prime}\right|^{1-\frac{k}{m}}, \quad \forall t, t^{\prime} \in(0, T), \quad 1 \leq k \leq m-1 ; \\
{\left[\partial^{m-1} w(t)-\partial^{m-1} w\left(t^{\prime}\right)\right]_{\theta, p, 1} \leq C_{2} M\left|t-t^{\prime}\right|^{1-\frac{m-1+\theta}{m}}, \quad \forall t, t^{\prime} \in(0 . T) .}
\end{gathered}
$$

(ii) Suppose that $F \in L^{\infty}\left([0, T) ; C_{B U}\left(R^{N}\right)\right)$ and there is a constant $M>0$ such that $\|F(t)\|_{\infty} \leq M$ for all $t \in[0, T)$. Then for any $0<\theta<1$ the function $w$ given in (4.1) belongs to $C\left([0, T) ; C_{B U}^{m-1+\theta}\left(R^{N}\right)\right) \cap C^{1 \#}\left([0, T) ; C_{B U}\left(R^{N}\right)\right)$. Moreover, for any $0 \leq k \leq$ $m-1\left(k \in Z_{+}\right)$and any $0<\theta<1$ there exist corresponding constants $C_{1}=C_{1}(k)>0$ and $C_{2}=C_{2}(\theta)>0$ independent of $T, M$ and $F$ such that (4.9)-(4.10) are valid with $p$ replaced with $\infty$.

Proof. We only prove the conclusion (i). The proof of the conclusion (ii) is similar and is thus omitted.

The assertion that $w \in C\left([0, T) ; B_{p 1}^{m-1+\theta}\left(R^{N}\right)\right) \cap C^{1 \#}\left([0, T) ; L^{p}\left(R^{N}\right)\right)$ follows directly from (4.9)-(4.11). To prove (4.9), without loss of generality we assume that $0<t<t^{\prime}$ and write

$$
w\left(t^{\prime}\right)-w(t)=\int_{t}^{t^{\prime}} S\left(t^{\prime}-\tau\right) F(\tau) \mathrm{d} \tau+\int_{0}^{t} \int_{t}^{t^{\prime}} \partial_{\sigma} S(\sigma-\tau) F(\tau) \mathrm{d} \sigma \mathrm{d} \tau
$$


Then by Theorem 3.3 we have

$$
\begin{aligned}
\left\|w\left(t^{\prime}\right)-w(t)\right\|_{p} & \leq C \int_{t}^{t^{\prime}}\|F(\tau)\|_{p} \mathrm{~d} \tau+C \int_{0}^{t} \int_{t}^{t^{\prime}}(\sigma-\tau)^{-1}\|F(\tau)\|_{p} \mathrm{~d} \sigma \mathrm{d} \tau \\
& \leq C M\left(t^{\prime}-t\right)+C M\left(t^{\prime} \ln t^{\prime}-t \ln t-\left(t^{\prime}-t\right) \ln \left(t^{\prime}-t\right)\right) .
\end{aligned}
$$

From this one easily gets (4.9). The proof of (4.10) and (4.11) is similar. Q. E. D.

Theorem 4.4 Let $1<p<\infty$ and $0<T \leq \infty$. Suppose that the assumption $\left(A_{1}\right)$ is satisfied and $F \in L^{p}\left((0, T) ; L^{p}\left(R^{N}\right)\right)$. Then the function $w$ given in (4.1) belongs to $L_{l o c}^{p}\left((0, T) ; W^{m, p}\left(R^{N}\right)\right) \cap W_{l o c}^{1, p}\left((0, T) ; L^{p}\left(R^{N}\right)\right)$. Moreover, there exists a constant $C>0$ independent of $T$ and $F$ such that

$$
\int_{0}^{T}\left\|\partial_{t} w(t)\right\|_{p}^{p} \mathrm{~d} t+\int_{0}^{T}\left\|\partial^{\alpha} w(t)\right\|_{p}^{p} \mathrm{~d} t \leq C \int_{0}^{T}\|F(t)\|_{p}^{p} \mathrm{~d} t
$$

Proof. We need only to prove that (4.12) is valid for sufficiently smooth $F$, say, $F \in$ $C_{0}^{\infty}\left((0, T) ; S\left(R^{N}\right)\right)$, because the general assertion then follows from a standard limitation procedure. Let

$$
\begin{gathered}
\hat{Q}(\xi, \tau)=\int_{0}^{\infty} \mathrm{e}^{-i t \tau} \tilde{Q}(\xi, t) \mathrm{d} t, \quad \hat{F}(\xi, \tau)=\int_{0}^{T} \mathrm{e}^{-i t \tau} \tilde{F}(\xi, t) \mathrm{d} t, \quad-\infty<\tau<\infty \\
A_{0}(\xi, \tau)=i \tau \hat{Q}(\xi, \tau), \quad A_{\alpha}(\xi, \tau)=(i \xi)^{\alpha} \hat{Q}(\xi, \tau)(|\alpha|=m) .
\end{gathered}
$$

Then since

$$
w(x, t)=(2 \pi)^{-N-1} \int_{-\infty}^{\infty} \int_{R^{N}} \hat{Q}(\xi, \tau) \hat{F}(\xi, \tau) \mathrm{e}^{i x \xi+i t \tau} \mathrm{d} \xi \mathrm{d} \tau
$$

we have

$$
\begin{gathered}
\partial_{t} w(x, t)=(2 \pi)^{-N-1} \int_{-\infty}^{\infty} \int_{R^{N}} A_{0}(\xi, \tau) \hat{F}(\xi, \tau) \mathrm{e}^{i x \xi+i t \tau} \mathrm{d} \xi \mathrm{d} \tau \\
\partial_{x}^{\alpha} w(x, t)=(2 \pi)^{-N-1} \int_{-\infty}^{\infty} \int_{R^{N}} A_{\alpha}(\xi, \tau) \hat{F}(\xi, \tau) \mathrm{e}^{i x \xi+i t \tau} \mathrm{d} \xi \mathrm{d} \tau, \quad|\alpha|=m .
\end{gathered}
$$

By making application of Lemma 3.1 one may verify that for arbitrary $k \in Z_{+}$and $\beta \in Z_{+}^{N}$,

$$
|\tau|^{k}|\xi|^{|\beta|}\left\|\partial_{\tau}^{k} \partial_{\xi}^{\beta} A_{0}(\xi, \tau)\right\| \leq C(k, \beta), \quad|\tau|^{k}|\xi|^{|\beta|}\left\|\partial_{\tau}^{k} \partial_{\xi}^{\beta} A_{\alpha}(\xi, \tau)\right\| \leq C(k, \beta) \quad(|\alpha|=m) .
$$

Thus by the varied Mihlin's multiplier theorem (see footnote on [23, p.290]), we get (4.12). Q. E. D.

To get the next theorem we need the following 
Lemma 4.5 Let $1 \leq p \leq \infty, 1 \leq r<\infty, s>0$ and $k, l \in Z_{+}$. Suppose that $k m+l>m+s$. Then for arbitrary $\varphi \in S\left(R^{N}\right)$ the following formulae hold:

$$
\begin{gathered}
{[\varphi]_{s, p, r} \sim \sum_{|\alpha|=l}\left(\int_{0}^{\infty}\left(\sigma^{k+\frac{l-s}{m}}\left\|\partial_{\sigma}^{k} \partial^{\alpha} S(\sigma) \varphi\right\|_{p}\right)^{r} \mathrm{~d} \sigma / \sigma\right)^{\frac{1}{r}},} \\
{[\varphi]_{s, p, \infty} \sim \sum_{|\alpha|=l} \sup _{\sigma>0}\left(\sigma^{k+\frac{l-s}{m}}\left\|\partial_{\sigma}^{k} \partial^{\alpha} S(\sigma) \varphi\right\|_{p}\right) .}
\end{gathered}
$$

The proof of this lemma is similar to that of [26, Proposition 7' and Lemma 4', Chapter V, p.151-152]. To save pages, we omit its proof here.

We also need the following

Lemma 4.6 Suppose that $\theta>1, a \geq 0$ and $b \geq 0$. Then there exists constant $C>0$ such that for any $t>0$ and $\sigma>0$,

$$
\int_{0}^{t}(t+\sigma-\tau)^{-\theta}(1+t+\sigma-\tau)^{-a}(1+\tau)^{-b} \mathrm{~d} \tau \leq\left\{\begin{array}{l}
C \sigma^{1-\theta}(1+t)^{-\min (1+a, b)}, \text { if } b \neq 1 \text { or } b=1 \text { and } a>0 \\
C \sigma^{1-\theta}(1+t)^{-1} \ln (2+t), \text { if } b=1 \text { and } a=0 .
\end{array}\right.
$$

proof. First assume that $0<t \leq 1$. Then

$$
\int_{0}^{t}(t+\sigma-\tau)^{-\theta}(1+t+\sigma-\tau)^{-a}(1+\tau)^{-b} \mathrm{~d} \tau \leq \int_{0}^{t}(t+\sigma-\tau)^{-\theta} \mathrm{d} \tau \leq \frac{1}{\theta-1} \sigma^{1-\theta} .
$$

Next assume that $t>1$. Then

$$
\int_{0}^{t}(t+\sigma-\tau)^{-\theta}(1+t+\sigma-\tau)^{-a}(1+\tau)^{-b} \mathrm{~d} \tau=\int_{0}^{\frac{t}{2}}+\int_{\frac{t}{2}}^{t} .
$$

We have

$$
\begin{aligned}
& \int_{0}^{\frac{t}{2}}(t+\sigma-\tau)^{-\theta}(1+t+\sigma-\tau)^{-a}(1+\tau)^{-b} \mathrm{~d} \tau \\
& \leq(\sigma+t / 2)^{-\theta}(1+\sigma+t / 2)^{-a} \int_{0}^{\frac{t}{2}}(1+\tau)^{-b} \mathrm{~d} \tau \\
& \leq \sigma^{1-\theta}(t / 2)^{-1}(1+t / 2)^{-a} \int_{0}^{\frac{t}{2}}(1+\tau)^{-b} \mathrm{~d} \tau \\
& \text { (because } \left.(\sigma+t / 2)^{-\theta}=(\sigma+t / 2)^{1-\theta}(\sigma+t / 2)^{-1} \text { and } 1-\theta<0\right) \\
& \leq \begin{cases}C \sigma^{1-\theta}(1+t)^{-(1+a)}, & \text { if } b>1 ; \\
C \sigma^{1-\theta}(1+t)^{-(1+a)} \ln (2+t), & \text { if } b=1 ; \\
C \sigma^{1-\theta}(1+t)^{-(a+b)}, & \text { if } b<1 ;\end{cases}
\end{aligned}
$$




$$
\int_{0}^{\frac{t}{2}}(t+\sigma-\tau)^{-\theta}(1+t+\sigma-\tau)^{-a}(1+\tau)^{-b} \mathrm{~d} \tau \leq(1+t / 2)^{-b} \int_{0}^{t}(t+\sigma-\tau)^{-\theta} \mathrm{d} \tau \leq C \sigma^{1-\theta}(1+t)^{-b} .
$$

Combining (4.16)-(4.19) together we obtain (4.15). Q. E. D.

Theorem 4.7 Let assumptions and notation be as in Theorem 4.2. Then $w \in$ $L^{\infty}\left((0, T) ; B_{p \infty}^{m}\left(R^{N}\right) \cap B_{q \infty}^{m}\left(R^{N}\right)\right)$ and there exists constant $C=C(p, q)>0$ independent of $T, M$ and $F$ such that for all $t>0$,

$$
[w(t)]_{m, q, \infty} \leq \begin{cases}C M(1+t)^{-\min \left(1+\frac{N}{m}\left(\frac{1}{p}-\frac{1}{q}\right), \kappa\right),}, & \text { if } \kappa \neq 1 \text { or } \kappa=1 \text { and } p \neq q \\ C M(1+t)^{-1} \ln (2+t), & \text { if } \kappa=1 \text { and } p=q .\end{cases}
$$

Proof. Take an integer $l$ such that $l>2 m$. By Lemma 4.5 we have

$$
[w(t)]_{m, q, \infty} \leq C \sup _{\sigma>0} \sigma^{\left(\frac{l}{m}-1\right)} \sum_{|\alpha|=l}\left\|\partial^{\alpha} S(\sigma) w(t)\right\|_{q} .
$$

Since $\partial^{\alpha} S(\sigma) w(t)=\int_{0}^{t} \partial^{\alpha} S(\sigma+t-\tau) F(\tau) \mathrm{d} \tau$, by (3.9), Theorem 3.3, we get

$$
\sum_{|\alpha|=l}\left\|\partial^{\alpha} S(\sigma) w(t)\right\|_{q} \leq C M \int_{0}^{t}(t+\sigma-\tau)^{-\frac{l}{m}}(1+t+\sigma-\tau)^{-\frac{N}{m}\left(\frac{1}{p}-\frac{1}{q}\right)}(1+\tau)^{-\kappa} \mathrm{d} \tau .
$$

Thus by making application of Lemma 4.6 we get the desired conclusion. Q. E. D.

\section{The Proofs of Theorem 2.1 and 2.2}

In this section we present the proofs of Theorem 2.1 and 2.2. The method we will use is to approximate the function $F$ which does not satisfy Lipchitz condition with a series of smooth functions. The crucial step is to prove that the corresponding series of solutions converges to a solution of the problem (1.1), which will be fulfilled with the aid of the estimates established in the above two sections.

Proof of Theorem 2.1: We first assume that $F$ is locally Lipchitz continuous. Let

$$
A=\|\varphi\|_{m-1, \infty}, \quad C_{0}=\sup _{t>0} \int_{R^{N}}\|Q(x, t)\| \mathrm{d} x .
$$

We now prove the following two assertions:

(i) There exists $T_{0}>0$ such that the problem (1.1) has a weak solution $u \in C\left(\left[0, T_{0}\right]\right.$; $\left.C_{B U}^{m-1}\left(R^{N}\right)\right)$ such that

$$
\left|\|u \mid\| \equiv \sup _{0 \leq t \leq T_{0}}\|u(t)\|_{m-1, \infty} \leq A C_{0}+1\right.
$$


(ii) If we denote by $T^{*}$ the supremum of all $T_{0}>0$ such that $(i)$ is valid, then either $T^{*}=\infty$ or $T^{*}<\infty$ and

$$
\limsup _{t \rightarrow T^{*}-0}\|u(t)\|_{m-1, \infty}=A C_{0}+1
$$

Let $L$ and $M$ be respectively the Lipchitz constant and maximal value of $F(w)$ on the closed ball $|w| \leq A C_{0}+1$, and denote by $\mathcal{F}$ the mapping defined by

$$
\mathcal{F} u(t)=S(t) \varphi+\int_{0}^{t} S(t-\tau) F\left(u(\tau), \partial u(\tau), \cdots, \partial^{m-1} u(\tau)\right) \mathrm{d} \tau .
$$

By utilizing (3.8) of Theorem 3.3 to the first term on the right side and (3.7) to the integrand we see that for arbitrary $T_{0}>0$ and arbitrary $u \in C\left(\left[0, T_{0}\right] ; C_{B U}^{m-1}\left(R^{N}\right)\right)$ satisfying $|\|u \mid\| \leq$ $A C_{0}+1$, we have ${ }^{1}$

$$
\begin{aligned}
\sum_{|\alpha| \leq m-1}\left\|\partial^{\alpha} \mathcal{F} u(t)\right\|_{\infty} & \leq C_{0} \sum_{|\alpha| \leq m-1}\left\|\partial^{\alpha} \varphi\right\|_{\infty}+C M \sum_{|\alpha| \leq m-1} \int_{0}^{t}(t-\tau)^{-\frac{|\alpha|}{m}} \mathrm{~d} \tau \\
& \leq A C_{0}+C M\left(T_{0}+T_{0}^{1-\frac{1}{m}}+\cdots+T_{0}^{\frac{1}{m}}\right), \quad \forall t \in\left[0, T_{0}\right],
\end{aligned}
$$

where $C$ represents positive constant depending only on $m, n, N$ and the coefficients of $P\left(\partial_{x}\right)$. Therefore, if $T_{0}>0$ is taken sufficiently small, then $\mathcal{F}$ maps the closed ball $|\|u \mid\| \leq$ $A C_{0}+1$ in $C\left(\left[0, T_{0}\right] ; C_{B U}^{m-1}\left(R^{N}\right)\right)$ into itself. Similarly, by making application of (3.7) of Theorem 3.3 and the Lipchitz continuity of $F$, we can prove that for $u, v \in C\left(\left[0, T_{0}\right] ; C_{B U}^{m-1}\left(R^{N}\right)\right)$ if they satisfy $\left|\left\|u\left|\left\|\leq A C_{0}+1,\left|\|v \mid\| \leq A C_{0}+1\right.\right.\right.\right.\right.$, then

$$
\sum_{|\alpha| \leq m-1}\left\|\partial^{\alpha} \mathcal{F} u(t)-\partial^{\alpha} \mathcal{F} v(t)\right\|_{\infty} \leq C L\left(T_{0}+T_{0}^{1-\frac{1}{m}}+\cdot+T_{0}^{\frac{1}{m}}\right)\left|\|u-v \mid\|, \quad \forall t \in\left[0, T_{0}\right] .\right.
$$

Thus by taking $T_{0}>0$ sufficiently small, we see that $\mathcal{F}$ is a contraction mapping. Consequently, $\mathcal{F}$ has a fixed point for such $T_{0}>0$. It is obvious that this fixed point is a weak solution of the problem (1.1) on $R^{N} \times\left[0, T_{0}\right]$. The assertion $(i)$ is proved. To prove (ii) we assume the contrary, i.e., $T^{*}<\infty$ and

$$
\limsup _{t \rightarrow T^{*}-0}\|u(t)\|_{m-1, \infty}<A C_{0}+1 .
$$

Since $\sup _{0 \leq t<T^{*}}\|u(t)\|_{\infty} \leq A C_{0}+1$, we have

$$
\left\|F\left(u(t), \partial u(t), \cdots, \partial^{m-1} u(t)\right)\right\|_{\infty} \leq M, \quad \forall t \in\left[0, T^{*}\right),
$$

Thus by applying (3.18) of Theorem 3.7 and (4.9)-(4.10) of Theorem 4.3 to the integral equation satisfied by $u$ we see that $u(t)$ converges in $C_{B U}^{m-1}\left(R^{N}\right)$ as $t \rightarrow T^{*}-0$. Let $\psi$ be the limit function. Then (5.1) implies $\|\psi\|_{m-1, \infty}<A C_{0}+1$. By repeating the arguement

\footnotetext{
${ }^{1}$ Remark: we note that the constant $C$ appearing in (3.8) can be taken to be $C_{0}$.
} 
we have just made, we get another number $T_{1}>0$ such that the problem (1.1) has a weak solution, which we denote as $v$, on $R^{N} \times\left[0, T_{1}\right]$ such that $v \in C\left(\left[0, T_{1}\right] ; C_{B U}^{m-1}\left(R^{N}\right)\right)$ and $\left|\|v \mid\| \leq A C_{0}+1\right.$ (with obvious modification on the definition of $\left.|\|\cdot \mid\|\right)$ ). Now we define $u(t)=v\left(t-T^{*}\right)$ for $t \in\left[T^{*}, T^{*}+T_{1}\right]$. Then $u \in C\left(\left[0, T^{*}+T_{1}\right] ; C_{B U}^{m-1}\left(R^{N}\right)\right),\left|\|u \mid\| \leq A C_{0}+1\right.$ (with obvious modification on $\left|\|\cdot \mid\|\right.$ ) and it is a weak solution of (1.1) on $R^{N} \times\left[0, T^{*}+T_{1}\right]$. This is contrary to the assumption on $T^{*}$. Hence the assertion $(i i)$ is also true.

Next we assume that $F$ is a general continuous function. Let $A$ and $C_{0}$ be as above and

$$
M=\sup _{|w| \leq A C_{0+2}}|F(w)| .
$$

Take a scalar function $\phi \in C_{0}^{\infty}\left(R^{N_{0}}\right)$ (where $N_{0}$ represents the dimension of the vector $\left.w\right)^{2}$ such that $0 \leq \phi \leq 1, \operatorname{supp} \phi \subset\left\{w \in R^{N_{0}}:|w| \leq 1\right\}$ and $\int_{R^{N_{0}}} \phi(w) \mathrm{d} w=1$. Define for each $\varepsilon \in(0,1)$ a function $F_{\varepsilon}$ on $R^{N_{0}}$ as follows:

$$
F_{\varepsilon}(w)=\frac{1}{\varepsilon^{N}} \int_{R^{N_{0}}} F\left(w^{\prime}\right) \phi\left(\frac{w-w^{\prime}}{\varepsilon}\right) \mathrm{d} w^{\prime}, \quad \forall w \in R^{N_{0}} .
$$

Obviously, $F_{\varepsilon}$ belongs to $C^{\infty}\left(R^{N_{0}}\right)$, converges uniformly on any compact subset of $R^{N_{0}}$ to $F$ as $\varepsilon \rightarrow 0$ and satisfies

$$
\left|F_{\varepsilon}(w)\right| \leq M, \quad \text { for }|w| \leq A C_{0}+1, \quad \forall \varepsilon \in(0,1) .
$$

Now let us consider the problem

$$
\left\{\begin{array}{l}
\partial_{t} u=P\left(\partial_{x}\right) u+F_{\varepsilon}\left(u, \partial u, \cdots, \partial_{x}^{m-1} u\right), \text { in } R^{N} \times(0, \infty), \\
\left.u\right|_{t=0}=\varphi, \text { on } R^{N}
\end{array}\right.
$$

By virtue of the conclusion we have just proved, there exists for each $\varepsilon \in(0,1)$ a corresponding $T_{\varepsilon}^{*} \in(0, \infty]$ such that the above problem has a weak solution $u=u_{\varepsilon}$ on $R^{N} \times\left[0, T_{\varepsilon}^{*}\right)$ satisfying the following two conditions:

(iii) $u_{\varepsilon} \in C\left([0, T] ; C_{B U}^{m-1}\left(R^{N}\right)\right)$ for any $T \in\left(0, T_{\varepsilon}^{*}\right)$, and

$$
\sup _{0 \leq t \leq T}\left\|u_{\varepsilon}(t)\right\|_{m-1, \infty} \leq A C_{0}+1, \quad \forall T \in\left(0, T_{\varepsilon}^{*}\right) ;
$$

(iv) if $T_{\varepsilon}^{*}<\infty$, then

$$
\limsup _{t \rightarrow T_{\varepsilon}^{*}-0}\left\|u_{\varepsilon}(t)\right\|_{m-1, \infty}=A C_{0}+1 .
$$

${ }^{2}$ Remark: $N_{0}=\left(\begin{array}{c}N-1 \\ N-1\end{array}\right)+\left(\begin{array}{c}N \\ N-1\end{array}\right)+\cdots+\left(\begin{array}{c}N+m-2 \\ N-1\end{array}\right)$. 
We now prove that there exists $T_{0}>0$ such that $T_{\varepsilon}^{*}>T_{0}$ for all $\varepsilon \in(0,1)$. In fact, from (iii) and (5.3) we see that

$$
\left\|F_{\varepsilon}\left(u_{\varepsilon}(t), \partial u_{\varepsilon}(t), \cdots, \partial^{m-1} u_{\varepsilon}(t)\right)\right\|_{\infty} \leq M, \quad \forall t \in\left[0, T_{\varepsilon}^{*}\right), \quad \forall \varepsilon \in(0,1) .
$$

Thus by applying (3.8) and (3.7) of Theorem 3.3 once again to the equivalent integral equation obtained from $\left(P_{\varepsilon}\right)$ we get

$$
\begin{aligned}
\sum_{|\alpha| \leq m-1}\left\|\partial^{\alpha} u_{\varepsilon}(t)\right\|_{\infty} & \leq C_{0} \sum_{|\alpha| \leq m-1}\left\|\partial^{\alpha} \varphi\right\|_{\infty}+C M \sum_{|\alpha| \leq m-1} \int_{0}^{t}(t-\tau)^{-\frac{|\alpha|}{m} \mathrm{~d} \tau} \\
& \leq A C_{0}+C M\left(t+t^{1-\frac{1}{m}}+\cdots+t^{\frac{1}{m}}\right), \quad \forall t \in\left[0, T_{\varepsilon}^{*}\right),
\end{aligned}
$$

where $C$ represents constant depending only on $m, n, N$ and the coefficients of $P\left(\partial_{x}\right)$. Now take $T_{0}>0$ sufficiently small such that

$$
C M\left(T_{0}+T_{0}^{1-\frac{1}{m}}+\cdots+T_{0}^{\frac{1}{m}}\right)<1 .
$$

Then we have

$$
\sup _{0 \leq t<\min \left(T_{0}, T_{\varepsilon}^{*}\right)}\left\|u_{\varepsilon}(t)\right\|_{m-1, \infty}<A C_{0}+1 .
$$

By virtue of $(i v)$, we conclude from this estimate that $T_{\varepsilon}^{*}>T_{0}$. Our assertion is proved. Consequently, $u_{\varepsilon}$ is well-defined on $R^{N} \times\left[0, T_{0}\right]$ for every $\varepsilon \in(0,1)$.

We are now going to prove that there is a sequence of numbers $\left\{\varepsilon_{j}\right\}_{j=1}^{\infty} \subset(0,1)$ satisfying $\lim _{j \rightarrow \infty} \varepsilon_{j}=0$ such that the corresponding sequence of functions $\left\{u_{\varepsilon_{j}}\right\}_{j=1}^{\infty}$ satisfies the following two conditions:

(v) for any compact subset $\bar{\Omega} \subset R^{N},\left\{u_{\varepsilon_{j}}\right\}_{j=1}^{\infty}$ converges in $C\left(\left[0, T_{0}\right] ; C^{m-2}(\bar{\Omega})\right)$;

(vi) for any compact subset $\bar{\Omega} \subset R^{N}$ and any $\delta \in\left(0, T_{0}\right),\left\{u_{\varepsilon_{j}}\right\}_{j=1}^{\infty}$ converges in $C\left(\left[\delta, T_{0}\right] ; C^{m-1}(\bar{\Omega})\right)$.

For this purpose we take arbitrarily a number $\theta \in(0,1)$ and prove that $\left\{u_{\varepsilon}: 0<\varepsilon<1\right\}$ is bounded in $C^{\frac{1-\theta}{m}}\left(\left[0, T_{0}\right] ; C^{m-2+\theta}\left(R^{N}\right)\right) \cap C^{\frac{1-\theta}{m}}\left(\left[\delta, T_{0}\right] ; C^{m-1+\theta}\left(R^{N}\right)\right)$ for arbitrary $\delta \in$ $\left(0, T_{0}\right)$. In fact, since

$$
u_{\varepsilon}(t)=S(t) \varphi+\int_{0}^{t} S(t-\tau) F_{\varepsilon}\left(u_{\varepsilon}(\tau), \partial u_{\varepsilon}(\tau), \cdots, \partial^{m-1} u_{\varepsilon}(\tau)\right) \mathrm{d} \tau,
$$

by making application of (3.20) and (3.21) in Theorem 3.7 and (4.9)-(4.11) in Theorem 4.3 we see that for all $t, t^{\prime} \in\left[0, T_{0}\right]$,

$$
\begin{gathered}
\left\|\partial^{\alpha} u_{\varepsilon}(t)-\partial^{\alpha} u_{\varepsilon}\left(t^{\prime}\right)\right\|_{\infty} \leq A C\left|t-t^{\prime}\right|^{1-\frac{|\alpha|+1}{m}}+C M\left|t-t^{\prime}\right|^{1-\frac{|\alpha|}{m}}, \quad \forall|\alpha| \leq m-2 ; \\
{\left[\partial^{\alpha} u_{\varepsilon}(t)-\partial^{\alpha} u_{\varepsilon}\left(t^{\prime}\right)\right]_{\theta} \leq A C\left|t-t^{\prime}\right|^{1-\frac{m-1+\theta}{m}}+C M\left|t-t^{\prime}\right|^{1-\frac{m-2+\theta}{m}}, \quad \forall|\alpha|=m-2,}
\end{gathered}
$$


where $[\cdot]_{\theta}$ represent the Hölder continuity modulus of functions. From the above estimates we see that $\left\{u_{\varepsilon}: 0<\varepsilon<1\right\}$ is bounded in $C^{\frac{1-\theta}{m}}\left(\left[0, T_{0}\right] ; C^{m-2+\theta}\left(R^{N}\right)\right)$. Similarly, by making application of (3.18) and (3.19) in Theorem 3.7 and, again, (4.9)-(4.11) in Theorem 4.3, we can prove that $\left\{u_{\varepsilon}: 0<\varepsilon<1\right\}$ is also bounded in $C^{\frac{1-\theta}{m}}\left(\left[\delta, T_{0}\right] ; C^{m-1+\theta}\left(R^{N}\right)\right)$ for arbitrary $\delta \in\left(0, T_{0}\right)$. From these conclusions, by virtue of the compactness of the embedding mappings from $C^{\frac{1-\theta}{m}}\left(\left[0, T_{0}\right] ; C^{m-2+\theta}(\bar{\Omega})\right)$ to $C\left(\left[0, T_{0}\right] ; C^{m-2}(\bar{\Omega})\right)$ and from $C^{\frac{1-\theta}{m}}\left(\left[\delta, T_{0}\right] ; C^{m-1+\theta}(\bar{\Omega})\right)$ to $C\left(\left[\delta, T_{0}\right] ; C^{m-1}(\bar{\Omega})\right)$ for arbitrary compact subset $\bar{\Omega}$ of $R^{N}$, we can use the diagonal subsequence method to prove the existence of the sequence $\left\{\varepsilon_{j}\right\}_{j=1}^{\infty}$ such that the corresponding sequence of functions $\left\{u_{\varepsilon_{j}}\right\}_{j=1}^{\infty}$ satisfies the conditions $(v)$ and $(v i)$. More precisely, take arbitrarily a sequence of numbers $\left\{\delta_{j}\right\}_{j=1}^{\infty} \subset\left(0, T_{0}\right)$ such that it is strictly decreasing and $\lim _{j \rightarrow \infty} \delta_{j}=0$, and a sequence of compact subsets of $R^{N}$, say, $\left\{\bar{\Omega}_{j}\right\}_{j=1}^{\infty}$, such that

$$
\bar{\Omega}_{1} \subset \bar{\Omega}_{2} \subset \cdots \subset \bar{\Omega}_{j} \subset \bar{\Omega}_{j+1} \subset \cdots \text { and } \bigcup_{j=1}^{\infty} \bar{\Omega}_{j}=R^{N} .
$$

Then by first taking arbitrarily a sequence of numbers $\left\{\varepsilon_{j}^{\prime}\right\}_{j=1}^{\infty} \subset(0,1)$ strictly decreasing and converging to zero as the starting sequence, then inductively applying the abovementioned compact embedding results to $\bar{\Omega}=\bar{\Omega}_{j}$ and $\delta=\delta_{j}$ for each $j$, and finally taking the diagonal subsequence, we get the desired conclusion. To save pages we omit the details here.

Now let $u$ be the limit function of the sequence $\left\{u_{\varepsilon_{j}}\right\}_{j=1}^{\infty}$. Obviuosly, $\left\{\partial^{\alpha} u_{\varepsilon_{j}}\right\}_{j=1}^{\infty}$ converges to $\partial^{\alpha} u$ pointwisely on $\left(0, T_{0}\right] \times R^{N}$ for all $|\alpha| \leq m-1$. Besides, we have

$$
\begin{aligned}
& F_{\varepsilon_{j}}\left(u_{\varepsilon_{j}}, \partial u_{\varepsilon_{j}}, \cdots, \partial^{m-1} u_{\varepsilon_{j}}\right)-F\left(u, \partial u, \cdots, \partial^{m-1} u\right) \\
= & \left\{F_{\varepsilon_{j}}\left(u_{\varepsilon_{j}}, \partial u_{\varepsilon_{j}}, \cdots, \partial^{m-1} u_{\varepsilon_{j}}\right)-F\left(u_{\varepsilon_{j}}, \partial u_{\varepsilon_{j}}, \cdots, \partial^{m-1} u_{\varepsilon_{j}}\right)\right\} \\
& +\left\{F\left(u_{\varepsilon_{j}}, \partial u_{\varepsilon_{j}}, \cdots, \partial^{m-1} u_{\varepsilon_{j}}\right)-F\left(u, \partial u, \cdots, \partial^{m-1} u\right)\right\} .
\end{aligned}
$$

Since $\sum_{|\alpha| \leq m-1}\left\|\partial^{\alpha} u_{\varepsilon_{j}}(t)\right\|_{\infty} \leq A C_{0}+1$ for all $t \in\left(0, T_{0}\right]$ and all $j \in Z_{+} \backslash\{0\}$, by the uniform convergence of $F_{\varepsilon}$ to $F$ on compact subset of $R^{N_{0}}$ and the continuity of $F$, we conclude from the above equality that $F_{\varepsilon_{j}}\left(u_{\varepsilon_{j}}, \partial u_{\varepsilon_{j}}, \cdots, \partial^{m-1} u_{\varepsilon_{j}}\right)$ converges to $F\left(u, \partial u, \cdots, \partial^{m-1} u\right)$ pointwisely on $R^{N} \times\left(0, T_{0}\right]$. Therefore, by first taking $\varepsilon=\varepsilon_{j}$ in (5.4) and then letting $j \rightarrow \infty$ and employing Lebesque's dominated convergence theorem, we see that $u$ is a weak solution of the problem (1.1) on $R^{N} \times\left[0, T_{0}\right]$. Moreover, since $\sup _{0<t \leq T_{0}} \sum_{|\alpha| \leq m-1}\left\|\partial^{\alpha} u_{\varepsilon_{j}}(t)\right\|_{\infty} \leq$ $A C_{0}+1(j=1,2, \cdots)$, we have

$$
\sum_{|\alpha| \leq m-1}\left|\partial_{x}^{\alpha} u(x, t)\right| \leq A C_{0}+1, \quad \forall t \in\left(0, T_{0}\right], \quad \forall x \in R^{N}, \quad \forall \alpha \in Z_{+}^{N}(|\alpha| \leq m-1) .
$$

Consequently,

$$
\sup _{0<t \leq T_{0}}\left\|F\left(u(t), \partial u(t), \cdots, \partial^{m-1} u(t)\right)\right\|_{\infty} \leq M
$$


Thus by making application of Theorems $3.3,4.2,4.3$ and 4.7 to the integral equation satisfied by $u$ we get

$$
u \in C\left(\left[0, T_{0}\right] ; C_{B U}^{m-1}\left(R^{N}\right)\right) \cap L_{l o c}^{\infty}\left(\left(0, T_{0}\right] ; C_{B U}^{m *}\left(R^{N}\right)\right) \cap C^{1 \#}\left(\left(0, T_{0}\right] ; C_{B U}\left(R^{N}\right)\right) .
$$

To finish the proof of Theorem 2.1, there still remains (2.1) to be proved. Since its proof is similar to the proof of the corresponding conclusion for the case where $F$ is locally Lipchitz continuous, we omit it here. Q. E. D.

Proof of Theorem 2.2: The proof of this theorem is similar to that of Theorem 2.1 given above. Thus we only present the outline here; the details are left to the reader.

First we assume that $F$ is locally Lipchitz continuous. Then, due to the additional assumption $\left(A_{2}\right)$, we can prove by using (3.7) and (3.8) in Theorem 3.3 that for every $\varphi \in L^{1}\left(R^{N}\right) \cap C_{B U}^{m-1}\left(R^{N}\right)$ the following assertions are valid:

$(i)^{\prime}$ There exists $T_{0}>0$ such that the problem (1.1) has a solution $u \in C\left(\left[0, T_{0}\right]\right.$; $\left.W^{m-1,1}\left(R^{N}\right) \cap C_{B U}^{m-1}\left(R^{N}\right)\right)$ satisfying

$$
\left|\|u \mid\|^{\prime} \equiv \sup _{0 \leq t \leq T_{0}} \sum_{|\alpha| \leq m-1}\left(\left\|\partial^{\alpha} u(t)\right\|_{1}+\left\|\partial^{\alpha} u(t)\right\|_{\infty}\right) \leq A C_{0}+1\right.
$$

where $C_{0}$ is as before and

$$
A=\|\varphi\|_{m-1,1}+\|\varphi\|_{m-1, \infty}
$$

(ii) If we denote by $T^{*}$ the supremum of all $T_{0}>0$ such that $(i)^{\prime}$ is valid, then either $T^{*}=\infty$ or $T^{*}<\infty$ and

$$
\limsup _{t \rightarrow T^{*}-0}\left(\|u(t)\|_{m-1,1}+\|u(t)\|_{m-1, \infty}\right)=A C_{0}+1 .
$$

Next we assume that $F$ is a general continuous function. Without loss of generality we may assume that $F$ satisfies the following stronger assumption than $\left(A_{2}\right)$ :

$\left(A_{2}^{\prime}\right)$ there exists a constant $C>0$ such that for all $w \in R^{N_{0}},|F(w)| \leq C|w|$.

Indeed, since our discussion is made for fixed $\varphi$, we can substitute $F$ which does not satisfy this condition with another continuous function satifying this condition such that it is equal to $F$ on the region $|w| \leq A C_{0}+2$. Let $\phi$ be as before and

$$
G_{\varepsilon}(w)=\frac{1}{\varepsilon^{N}} \int_{\left|w^{\prime}\right| \geq 2 \varepsilon} F\left(w^{\prime}\right) \phi\left(\frac{w-w^{\prime}}{\varepsilon}\right) \mathrm{d} w^{\prime}, \quad \forall w \in R^{N_{0}} .
$$

We have

$$
G_{\varepsilon}(w)=F_{\varepsilon}(w)-\frac{1}{\varepsilon^{N}} \int_{\left|w^{\prime}\right|<2 \varepsilon} F\left(w^{\prime}\right) \phi\left(\frac{w-w^{\prime}}{\varepsilon}\right) \mathrm{d} w^{\prime}, \quad \forall w \in R^{N_{0}}
$$


where $F_{\varepsilon}$ is the function given in (5.2). From this relation and the assumption $\left(A_{2}^{\prime}\right)$ we see easily that $G_{\varepsilon}$ converges to $F$ uniformly on compact subset of $R^{N_{0}}$ as $\varepsilon \rightarrow 0$. Moreover, we have

$$
\left|G_{\varepsilon}(w)\right| \leq 2 C|w|, \quad \forall w \in R^{N_{0}}, \quad \forall \varepsilon \in(0,1),
$$

where $C$ is the constant appearing in the assumption $\left(A_{2}^{\prime}\right)$. In fact, it is obvious that $G_{\varepsilon}(w)=0$ for $|w|<\varepsilon$. For $|w| \geq \varepsilon$ we have

$$
\begin{aligned}
\left|G_{\varepsilon}(w)\right| & \leq \frac{C}{\varepsilon^{N}} \int_{\left|w^{\prime}\right| \geq 2 \varepsilon}\left|w^{\prime}\right| \phi\left(\frac{w-w^{\prime}}{\varepsilon}\right) \mathrm{d} w^{\prime} \\
& \leq \frac{C \varepsilon}{\varepsilon^{N}} \int_{R^{N_{0}}}\left|\frac{w^{\prime}-w}{\varepsilon}\right| \phi\left(\frac{w-w^{\prime}}{\varepsilon}\right) \mathrm{d} w^{\prime}+\frac{C|w|}{\varepsilon^{N}} \int_{R^{N_{0}}} \phi\left(\frac{w-w^{\prime}}{\varepsilon}\right) \mathrm{d} w^{\prime} \leq 2 C|w| .
\end{aligned}
$$

Now by applying the conclusions stated at the beginning, we get for each $\varepsilon \in(0,1)$ a number $T_{\varepsilon}^{*} \in(0, \infty]$ such that the problem $\left(P_{\varepsilon}\right)$ with $F_{\varepsilon}$ replaced by $G_{\varepsilon}$ has a weak solution $u=u_{\varepsilon}$ satisfying

$(i i i)^{\prime} u_{\varepsilon} \in C\left([0, T] ; W^{m-1,1}\left(R^{N}\right) \cap C_{B U}^{m-1}\left(R^{N}\right)\right)$ for any $T \in\left(0, T_{\varepsilon}^{*}\right)$, and

$$
\sup _{0 \leq t \leq T}\left(\left\|u_{\varepsilon}(t)\right\|_{m-1,1}+\left\|u_{\varepsilon}(t)\right\|_{m-1, \infty}\right) \leq A C_{0}+1, \quad \forall T \in\left(0, T_{\varepsilon}^{*}\right)
$$

$(i v)^{\prime}$ if $T_{\varepsilon}^{*}<\infty$, then

$$
\limsup _{t \rightarrow T_{\varepsilon}^{*}-0}\left(\left\|u_{\varepsilon}(t)\right\|_{m-1,1}+\left\|u_{\varepsilon}\right\|_{m-1, \infty}\right)=A C_{0}+1 .
$$

By employing the uniform estimates given in (5.5) and making similar arguement as at the corresponding step in the proof of Theorem 2.1, we conclude from these assertionss that there exists $T_{0}>0$ such that $T_{\varepsilon}^{*}>T_{0}$ for all $\varepsilon \in(0,1)$. Besides, by applying Corollaries 3.4, 3.6 and Theorems 3.7, 4.2 and 4.3 we can prove that $\left\{u_{\varepsilon}: 0<\varepsilon<1\right\}$ is bounded in

$$
C^{\frac{1-\theta}{m}}\left(\left[0, T_{0}\right] ; B_{11}^{m-2+\theta}\left(R^{N}\right) \cap C_{B U}^{m-2+\theta}\left(R^{N}\right)\right) \cap C^{\frac{1-\theta}{m}}\left(\left[\delta, T_{0}\right] ; B_{11}^{m-1+\theta}\left(R^{N}\right) \cap C_{B U}^{m-1+\theta}\left(R^{N}\right)\right)
$$

for arbitrary $\theta \in(0,1)$ and $\delta \in\left(0, T_{0}\right)$. Therefore, by using again the diagonal subsequence method we get a sequence of numbers $\left\{\varepsilon_{j}\right\}_{j=1}^{\infty} \subset(0,1)$ such that the corresponding sequence of functions $\left\{u_{\varepsilon_{j}}\right\}_{j=1}^{\infty}$ converges in

$$
C\left(\left[0, T_{0}\right] ; W^{m-2,1}(\bar{\Omega}) \cap C_{B U}^{m-2}(\bar{\Omega})\right) \cap C\left(\left[\delta, T_{0}\right] ; W^{m-1,1}(\bar{\Omega}) \cap C_{B U}^{m-1}(\bar{\Omega})\right)
$$

for arbitrary $\delta \in\left(0, T_{0}\right)$ and arbitrary compact subset $\bar{\Omega}$ of $R^{N}$. From the proof of Theorem 2.1 we see that the limit function $u$ is a weak solution of the problem (1.1) on $R^{N} \times\left[0, T_{0}\right]$. To see that $u$ is a strong solution, we note that by virtue of Fatou's lemma we have

$$
\|u(t)\|_{m-1,1} \leq \sup _{j}\left\{\left\|u_{\varepsilon_{j}}(t)\right\|_{m-1,1},\right.
$$


and by the pointwise convergence of $\partial^{\alpha} u_{\varepsilon_{j}}$ to $\partial^{\alpha} u$ we get

$$
\left|\partial_{x}^{\alpha} u(x, t)\right| \leq \sup _{j}\left\|\partial^{\alpha} u_{\varepsilon_{j}}(t)\right\|_{\infty}, \quad \forall t \in\left(0, T_{0}\right], \quad \forall x \in R^{N}, \quad \forall|\alpha| \leq m-1 .
$$

Therefore,

$$
\|u(t)\|_{m-1,1}+\|u(t)\|_{m-1, \infty} \leq A C_{0}+1, \quad \forall t \in\left(0, T_{0}\right] .
$$

Consequently, by the assumption $\left(A_{2}^{\prime}\right)$ we get

$$
\left\|F\left(u(t), \partial u(t), \cdots, \partial^{m-1} u(t)\right)\right\|_{1}+\left\|F\left(u(t), \partial u(t), \cdots, \partial^{m-1} u(t)\right)\right\|_{\infty} \leq C, \quad \forall t \in\left(0, T_{0}\right] .
$$

From this estimate, by making application of Corollary 3.4, Theorems 3.7, 3.8, 4.2, 4.3 and 4.7 to the equivalent integral equation satisfied by $u$, we finally get

$$
\begin{aligned}
& u \in C\left(\left[0, T_{0}\right] ; W^{m-1,1}\left(R^{N}\right) \cap C_{B U}^{m-1}\left(R^{N}\right)\right) \cap L_{l o c}^{p}\left(\left[0, T^{*}\right) ; W^{m, p}\left(R^{N}\right)\right) \\
& \cap W_{l o c}^{1, p}\left(\left[0, T^{*}\right) ; L^{p}\left(R^{N}\right)\right), \quad \forall p \in(1, m] .
\end{aligned}
$$

Hence $u$ is actually a strong solution of (1.1) on $R^{N} \times\left[0, T_{0}\right]$.

The remaining conclusion in Theorem 2.2 , i.e., the validity of $(2.2)$, can be proved by using similar method as in the case where $F$ is Lipchitz continuous. Q. E. D.

\section{The Proof of Theorem 2.3}

Proof of Theorem 2.3: By Theorem 2.2, we only need to prove that there exists $\delta>0$ such that for any $\varphi \in W^{m-1,1}\left(R^{N}\right) \cap C_{B U}^{m-1}\left(R^{N}\right)$ satisfying (2.6) the corresponding solution $u$ of (1.1) satisfies

$$
\sup _{0 \leq t<T}\left(\|u(\cdot, t)\|_{m-1,1}+\|u(\cdot, t)\|_{m-1, \infty}\right) \leq M
$$

when it exists on $R^{N} \times[0, T)$, where $T>0$ is an arbitrary finite number and $M$ is the number appearing in the assumption $\left(A_{3}\right)$.

We first assume $\delta<M$. Then there must be a maximal $T_{0} \in(0, T]$ such that

$$
\|u(\cdot, t)\|_{m-1,1}+\|u(\cdot, t)\|_{m-1, \infty} \leq M
$$

for all $t \in\left[0, T_{0}\right)$. Let

$$
\begin{aligned}
f(t)= & \sum_{k=0}^{m-1}\left\{(1+t)^{\frac{k}{m}}\left\|\partial^{k} u(\cdot, t)\right\|_{1}+(1+t)^{\frac{k}{m}+\frac{N}{m}\left(1-\frac{1}{p_{k}}\right)}\left\|\partial^{k} u(\cdot, t)\right\|_{p_{k}}\right. \\
& \left.+(1+t)^{\min \left(\frac{k}{m}+\frac{N}{m}, \gamma\right)}\left\|\partial^{k} u(\cdot, t)\right\|_{\infty}\right\}, 0 \leq t<T_{0},
\end{aligned}
$$


where $\gamma$ is the number defined in (2.4). Then by the assumption $\left(A_{3}\right)$ we have

$$
\begin{aligned}
& \left\|F\left(u(\cdot, t), \partial u(\cdot, t), \cdots, \partial^{m-1} u(\cdot, t)\right)\right\|_{1} \\
& \leq C\left\{\|u(\cdot, t)\|_{p_{0}}^{p_{0}}+\|\partial u(\cdot, t)\|_{p_{1}}^{p_{1}}+\cdots+\left\|\partial^{m-1} u(\cdot, t)\right\|_{p_{m-1}}^{p_{m-1}}\right\} \\
& \leq C \sum_{k=0}^{m-1}(1+t)^{-\frac{(N+k) p_{k}-N}{m}}\left\{(1+t)^{\frac{k}{m}+\frac{N}{m}\left(1-\frac{1}{p_{k}}\right)}\left\|\partial^{k} u(\cdot, t)\right\|_{p_{k}}\right\}^{p_{k}} \\
& \leq C(1+t)^{-\gamma} \sum_{k=0}^{m-1} f(t)^{p_{k}} \\
& \leq C(1+t)^{-\gamma} \sum_{k=0}^{m-1}\left(\sup _{0 \leq t \leq t^{\prime}} f(t)\right)^{p_{k}}, \quad 0 \leq t \leq t^{\prime}<T_{0} ; \\
& \left\|F\left(u(\cdot, t), \partial u(\cdot, t), \cdots, \partial^{m-1} u(\cdot, t)\right)\right\|_{\infty} \\
& \leq C\left\{\|u(\cdot, t)\|_{\infty}^{p_{0}}+\|\partial u(\cdot, t)\|_{\infty}^{p_{1}}+\cdots+\left\|\partial^{m-1} u(\cdot, t)\right\|_{\infty}^{p_{m-1}}\right\} \\
& \leq C \sum_{k=0}^{m-1}(1+t)^{-p_{k} \min \left(\frac{N+k}{m}, \gamma\right)}\left\{(1+t)^{\min \left(\frac{N+k}{m}, \gamma\right)}\left\|\partial^{k} u(\cdot, t)\right\|_{\infty}\right\}^{p_{k}} \\
& \leq C(1+t)^{-\gamma} \sum_{k=0}^{m-1}\left(\sup _{0 \leq t \leq t^{\prime}} f(t)\right)^{p_{k}}, \quad 0 \leq t \leq t^{\prime}<T_{0} .
\end{aligned}
$$

Here $C$ is a constant independent of $T_{0}, \varphi$ and $u$. Since $\gamma>1$, by applying Corollary 3.4 and Theorem 4.2 to the equivalent integral equation satisfied by $u$ we get for all $0 \leq t \leq t^{\prime}<T_{0}$ and $0 \leq j \leq m-1$,

$$
\begin{gathered}
\left\|\partial^{j} u(\cdot, t)\right\|_{1} \leq C(1+t)^{-\min \left(\frac{j}{m}, \gamma\right)}\left\{\|\varphi\|_{m-1,1}+\|\varphi\|_{m-1, \infty}+\sup _{0 \leq t \leq t^{\prime}} \sum_{k=0}^{m-1} f(t)^{p_{k}}\right\}, \\
\left\|\partial^{j} u(\cdot, t)\right\|_{p_{j}} \leq C(1+t)^{-\min \left\{\frac{j}{m}+\frac{N}{m}\left(1-\frac{1}{p_{j}}\right), \gamma\right\}}\left\{\|\varphi\|_{m-1,1}+\|\varphi\|_{m-1, \infty}+\sup _{0 \leq t \leq t^{\prime}} \sum_{k=0}^{m-1} f(t)^{p_{k}}\right\}, \\
\left\|\partial^{j} u(\cdot, t)\right\|_{\infty} \leq C(1+t)^{-\min \left(\frac{j}{m}+\frac{N}{m}, \gamma\right)}\left\{\|\varphi\|_{m-1,1}+\|\varphi\|_{m-1, \infty}+\sup _{0 \leq t \leq t^{\prime}} \sum_{k=0}^{m-1} f(t)^{p_{k}}\right\} .
\end{gathered}
$$

Note that the hypothesis (2.3) implies $\gamma \geq \frac{j}{m}+\frac{N}{m}\left(1-\frac{1}{p_{j}}\right)$ for all $j=0,1, \cdots, m-1$. Thus we finally get

$$
f(t) \leq C\left\{\delta+\sup _{0 \leq t \leq t^{\prime}} \sum_{k=0}^{m-1} f(t)^{p_{k}}\right\}, \quad 0 \leq t \leq t^{\prime}<T_{0}
$$

where the constant $C>0$ is independent of $\delta$ (and hence $\varphi$ ), $u$ and $T_{0}$. Without loss of generality we may assume $C \geq 2$, for otherwise we can substitute it with 2 . Now let us consider the function

$$
g_{\delta}(s)=C\left(\delta+s^{p_{0}}+s^{p_{1}}+\cdots+s^{p_{m-1}}\right)-s, \quad 0 \leq s<\infty .
$$

Obviously, there exists $\delta_{1}>0$ such that when $0<\delta<\delta_{1}$, the region

$$
R_{\delta}=\left\{s: s \geq 0, g_{\delta}(s) \geq 0\right\}
$$

is disconnected, consisting of two disjoint closed intervals, with the left one in the form $\left[0, A_{\delta}\right]$ $\left(A_{\delta}=\right.$ the first nonnegative root of $g_{\delta}$ ) and the right one strenching to $\infty$. One may easily 
verify that $\lim _{\delta \rightarrow 0^{+}} A_{\delta}=0$ and $A_{\delta} \geq C \delta$ with $C$ as in (6.4). Indeed, the first statement follows from the fact that $g_{\delta}$ depends continuously on $\delta$ and $A_{\delta}=0$ when $\delta=0$; the second one follows from the fact that $g_{\delta}(s)>0$ for $0 \leq s<C \delta$. Therefore, there exists $\delta_{0} \in\left(0, \delta_{1}\right]$ such that $A_{\delta}<M$ for $0<\delta<\delta_{0}$. Take a such $\delta$ and fix it. This $\delta$ meets our need; namely, (6.1) is valid, or equivalently, $T_{0}=T$. In fact, (6.3) tells us that the function

$$
t^{\prime} \rightarrow \sup _{0 \leq t \leq t^{\prime}} f(t), \quad \forall t^{\prime} \in\left[0, T_{0}\right)
$$

takes value in $R_{\delta}$ for all $t^{\prime} \in\left[0, T_{0}\right)$. Since this function is continuous and takes the value $f(0) \leq 2 \delta \leq C \delta \leq A_{\delta}$ at $t^{\prime}=0$, we conclude that

$$
\sup _{0 \leq t \leq t^{\prime}} f(t) \leq A_{\delta} \quad \text { for all } 0 \leq t^{\prime}<T_{0}
$$

which especially implies

$$
\|u(\cdot, t)\|_{m-1,1}+\|u(\cdot, t)\|_{m-1, \infty} \leq A_{\delta}<M \quad \text { for all } 0 \leq t<T_{0} .
$$

From this we see that $T_{0}$ can not be the maximal number such that (6.2) is valid unless $T_{0}=T$. Our statement is proved.

Hence, by virtue of Theorem 2.2 we conclude that $u$ is global if $\varphi$ satisfies (2.6) with $\delta$ as above. Moreover, since $A_{\delta}$ is independent of $T,(6.4)$ tells us that for every $k=0,1, \cdots, m-1$ and all $t \in[0, \infty)$,

$$
\begin{gathered}
\left\|\partial^{k} u(\cdot, t)\right\|_{1} \leq A_{\delta}(1+t)^{-\frac{k}{m}}, \quad\left\|\partial^{k} u(\cdot, t)\right\|_{p_{k}} \leq A_{\delta}(1+t)^{-\left[\frac{k}{m}+\frac{N}{m}\left(1-\frac{1}{p_{k}}\right)\right]} \\
\left\|\partial^{k} u(\cdot, t)\right\|_{\infty} \leq A_{\delta}(1+t)^{-\min \left(\frac{k}{m}+\frac{N}{m}, \gamma\right)}
\end{gathered}
$$

From these estimates one may easily get (2.7) by applying (3.10), Theorem 3.3 and (4.7), Theorem 4.2. As for (2.8), it can also be easily obtained from the above estimates by applying Theorem 3.8 and Theorem 4.4. The proof of Theorem 2.3 is finished.

\section{References}

[1] A. Haraux, Nonlinear Evolution Equations-Global Behavior of Solutions, Springer-Verlag, New York, 1981.

[2] A. Pazy, Semigroups of Linear Operators and Applications to Partial Differential Equations, Springer-Verlag, New York, 1983.

[3] M. Roseau, Equations Differentielles, Masson, Paris, 1976.

[4] A. Pazy, A class of semilinear equations of evolution, Isreal J. Math., 20(1975), 23-36.

[5] J. H. Lightbourne and R. H. Martin, Relatively continuous nonlinear perturbations of analytic semigroups, Nonlinear Anal., 1(1977), 277-292.

[6] A. Friedman, Remarks on nonlinear parabolic equations, Applications of Nonlinear Partial Differential Equations in Mathematical Physics (Proc. of Symposia in Appl. Math., Vol.17), 3-23, Amer. Math. Soc., Providence, 1965. 
[7] H. Fujita, On the blowing up of the Cauchy problem for $u_{t}=\Delta u+u^{1+\alpha}$, J. Fac. Sci. Univ. Tokyo, Sect. I, 13(1966), 109-124.

[8] K. Kobayashi, T. Sirao and H. Tanaka, On the blowing up problem for semilinear heat equations, J. Math. Soc. Japan, 29(1977), 407-424.

[9] F. Weissler, Existence and nonexistence of global solutions for a semilinear heat equation, Israel J. Math., 38(1981), 29-40.

[10] G. Ponce, Global existence of small solutions to a class of nonlinear evolution equations, Nonlinear Anal., 9(1985), 399-418.

[11] Zheng Song-mu, Remarks on global existence for nonlinear parabolic equations, Nonlinear Anal., 10(1986), 107-114.

[12] A. Friedman and A. A. Lacey, Blow-up time for for solutions of nonlinear heat equation with small diffusion, SIAM J. Math. Anal., 18(1987), 711-721.

[13] M. Chipot and F. B. Weissler, Some blowup results for a nonlinear parabolic equation with a gradient term, SIAM J. Math. Anal., 20(1989), 886-907.

[14] P. Souplet, Finite time blow-up for a non-linear parabolic equation with a gradient term and applications, Math. Methods in Appl. Sci., 19(1996), 1317-1333.

[15] Cui Shangbin, Initial value problems for higher order nonlinear parabolic systems, Acta Math. Sci., Chinese Series, 15(1995), 209-216.

[16] Cui Shangbin, Initial value problems for higher order nonlinear parabolic systems possessing nonhomogeneous linear terms, Chinese Ann. Math., 16A(1995), 692-701.

[17] Cui Shangbin, Global solutions for initial value problems of higher order nonlinear parabolic systems possessing nonhomogeneous linear terms, Chinese Ann. Math., 17A(1996), 745-752.

[18] J. Aguirre and M. Escobedo, Source solutions for a convection diffusion problem: Global existence and blow-up, preprint.

[19] W. Liu, Singular solutions for a convection diffusion equation with absorption, J. Math. Anal. Appl., 163(1992), 200-219.

[20] M. Escobedo and M. A. Herrero, Boundedness and blow up for a semilinear reaction-diffusion system, J. Diff. Equa., 89(1991), 176-202.

[21] Cui Shangbin, Global behavior of solutions to a semilinear reaction-diffusion system, preprint.

[22] A. Friedman, Partial Differential Equations of Parabolic Type, Prentice-Hall, New Jersey, 1964.

[23] O. A. Ladyzenskaya, V. A. Solonnikov and N. N. Ural'ceva, Linear and Quasi-linear Equations of Parabolic Type (Trans. of Math. Monographs, Vol.23), Amer. Math. Soc., Providence, 1968.

[24] J. Bergh and J. Löfström, Interpolation Spaces, an Introduction, Springer-Verlag, New York, 1976.

[25] I. M. Gelfand and G. E. Silov, Generalized Functions, Vol.III, Moscov, 1958.

[26] E. M. Stein, Singular Integrals and Differentiability Properties of Functions, Princeton Univ. Press, Princeton, 1970. 